\title{
THE STABILITY OF WEAKLY COLLISIONAL PLASMAS WITH THERMAL AND COMPOSITION GRADIENTS
}

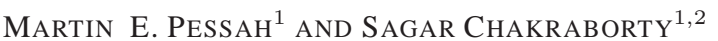 \\ ${ }^{1}$ Niels Bohr International Academy, Niels Bohr Institute, Blegdamsvej 17, DK-2100 Copenhagen $\emptyset$, Denmark; mpessah@nbi.dk \\ ${ }^{2}$ Department of Physics, Indian Institute of Technology, Kanpur, U.P.-208016, India; sagarc@iitk . ac . in \\ Draft version March 1, 2019
}

\begin{abstract}
Over the last decade, substantial efforts have been devoted to understanding the stability properties, transport phenomena, and long-term evolution of weakly collisional, magnetized plasmas which are stratified in temperature. The insights gained via these studies have led to a significant improvement of our understanding of the processes that determine the physical evolution and observational properties of the intracluster medium (ICM) permeating galaxy clusters. These studies have been carried out under the assumption that the ICM is a homogeneous medium. This, however, might not be a good approximation if heavy elements are able to sediment in the inner region of the galaxy cluster. Motivated by the need to obtain a more complete picture of the dynamical properties of the ICM, we analyze the stability of a weakly collisional, magnetized plane-parallel atmosphere which is stratified in both temperature and composition. This allows us to discuss for the first time the dynamics of weakly collisional environments where heat conduction, momentum transport, and ion-diffusion are anisotropic with respect to the direction of the magnetic field. We show that, depending on the relative signs and magnitudes of the gradients in the temperature and the mean molecular weight, the plasma can be subject to a wide variety of unstable modes which include modifications to the magnetothermal instability (MTI), the heat-flux-driven buoyancy instability (HBI), and overstable gravity modes previously studied in homogeneous media. We also find that there are new modes which are driven by heat conduction and particle diffusion. We discuss the astrophysical implications of our findings for a representative galaxy cluster where helium has sedimented. Our findings suggest that the core insulation that results from the magnetic field configurations that arise as a natural consequence of the HBI, which would be MTI stable in a homogeneous medium, could be alleviated if the mean molecular weight gradient is steep enough, i.e., $(\nabla \mu) / \mu>(\nabla T) / T$. This study constitutes a first step toward understanding the interaction between magnetic turbulence and the diffusion of heavy elements, and its consequences for the long-term evolution and observational signatures of the ICM in galaxy clusters.
\end{abstract}

Subject headings: galaxies: clusters: intracluster medium — instabilities — magnetohydrodynamics

\section{INTRODUCTION}

Despite the fact that magnetic fields in galaxy clusters are too weak to be mechanically important, they can play a fundamental role in the dynamical stability of the dilute gas by channeling the transport of heat, momentum, and particles. The weakly collisional character of the hot intracluster medium (ICM), which is generically characterized by stable entropy gradients according to Schwarzschild's criterion (Piffaretti et al. 2005; Cavagnolo et al. 2009), enables the action of magnetic instabilities that are sensitive to temperature gradients (Balbus 2000, 2004). In particular, the magnetothermal instability (MTI) exhibits the fastest growing modes when magnetic field lines are orthogonal to a temperature gradient parallel to the gravitational field (Balbus 2001), whereas the heat-flux-driven buoyancy instability (HBI) does so when magnetic field lines are parallel to a temperature gradient which is anti-parallel to the gravitational field (Ouataert 2008)

While the landscape of thermal instabilities that render homogeneous, dilute plasmas unstable has been well explored (Kunz 2011), and even extended to account for the effects of cosmic rays (Chandran \& Dennis 2006; Sharma et al. 2010), very little is known about the effects that composition gradients can have on the stability of the dilute ICM. If magnetic fields do not prevent the efficient diffusion of ions (Narayan \& Medvedev 2001; Chuzhoy \& Nusser 2003; Chuzhoy \& Loeb 2004) then the gradients in mean molecular weight can be as important as the gradients in tempera- ture (see Section 7 and Oin \& Wu 2000; Peng \& Nagai 2009; Shtykovskiy \& Gilfanov 2010; Bulbul et al. 2011) and provide another source of free energy to feed instabilities. In order to obtain a more complete picture of the stability properties of the ICM, it is thus important to relax the assumption of a homogeneous medium.

As a first step toward understanding the role of composition gradients in the stability of dilute plasmas, such as the ICM, we analyze the stability of a weakly magnetized plane-parallel atmosphere where magnetic fields play a key role by channeling the conduction of heat, transport of momentum, and the diffusion of ions. Our analysis generalizes previous studies on the MTI (Balbus 2001), the HBI (Quataert 2008), and overstable gravity modes (Balbus \& Reynolds 2010), and reveals the subtle roles played by the temperature and the composition gradients in determining the stability of the plasma.

The outline of the paper is as follows. In Section 2 we describe the plasma model for a dilute binary mixture of ions. In Section 3 we perform the linear mode analysis and we obtain the general dispersion relation that governs the linear dynamics of a weakly magnetized medium which is stratified in temperature and composition. We analyze in detail the stability of the plasma in the regimes where conduction across a given scale is, respectively, fast and slow compared to the dynamical timescale in Sections 4 and 5 In Section 6 we describe the physics driving the most relevant instabilities. We discuss the astrophysical implications of this study in Section 7. 


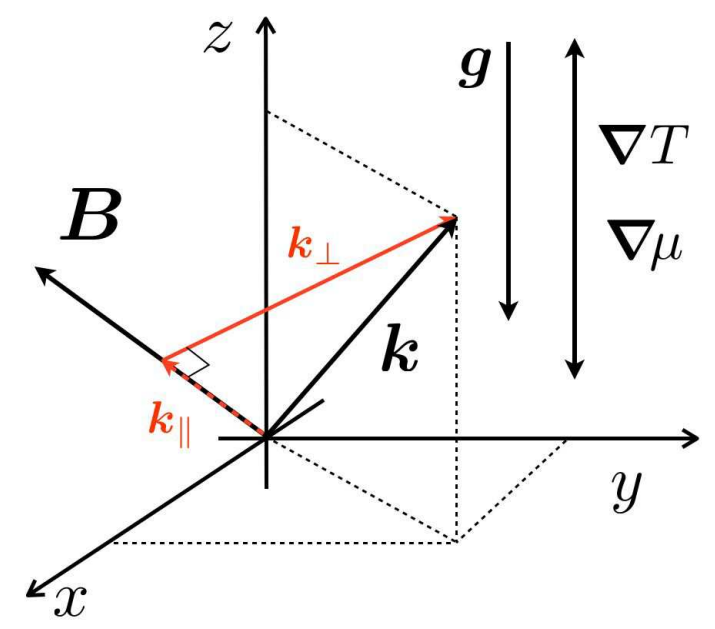

Figure 1. Schematic representation of the geometry involved in the stability analysis of the dilute, magnetized plane-parallel atmosphere with density, temperature, and composition gradients. The symbols $\|$ and $\perp$ label the directions parallel and perpendicular to the magnetic field, which is assumed to lie on the $x-z$ plane, without loss of generality.

\section{MODEL FOR THE MULTI-COMPONENT, DILUTE ATMOSPHERE}

\subsection{General Considerations for the Plasma Model}

In order to highlight the physical phenomena that emerge when composition gradients are accounted for, we focus our attention on a dilute binary mixture (e.g., hydrogen and he-

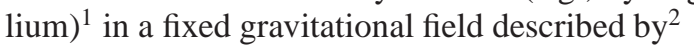

$$
\begin{aligned}
& \frac{\partial \rho}{\partial t}+\nabla \cdot(\rho \boldsymbol{v})=0, \\
& \frac{\partial}{\partial t}(\rho \boldsymbol{v})+\nabla \cdot\left(\rho \boldsymbol{v} \boldsymbol{v}+\mathrm{P}+\frac{B^{2}}{8 \pi} \mathrm{I}-\frac{B^{2}}{4 \pi} \hat{\boldsymbol{b}} \hat{\boldsymbol{b}}\right)=\rho \boldsymbol{g}, \\
& \frac{\partial \boldsymbol{B}}{\partial t}=\nabla \times(\boldsymbol{v} \times \boldsymbol{B}), \\
& \frac{P}{\gamma-1} \frac{d}{d t}\left(\ln P \rho^{-\gamma}\right)=\left(p_{\perp}-p_{\|}\right) \frac{d}{d t} \ln \frac{B}{\rho^{2 / 3}}-\boldsymbol{\nabla} \cdot \boldsymbol{Q}_{\mathrm{s}}, \\
& \frac{d c}{d t}=-\boldsymbol{\nabla} \cdot \boldsymbol{Q}_{\mathrm{c}} .
\end{aligned}
$$

Here, the Lagrangian and Eulerian derivatives are related via $d / d t \equiv \partial / \partial t+\boldsymbol{v} \cdot \nabla, \rho$ is the mass density, $\boldsymbol{v}$ is the fluid velocity, $\boldsymbol{g}$ is the gravitational acceleration, $\gamma$ is the adiabatic index, and I stands for the $3 \times 3$ identity matrix. The symbols $\perp$ and $\|$ refer respectively to the directions perpendicular and parallel to the magnetic field $\boldsymbol{B}$, see Figure 1 whose direction is given by the versor $\hat{\boldsymbol{b}} \equiv \boldsymbol{B} / B=\left(b_{x}, 0, b_{z}\right)$. The first term on the right-hand side of Equation (4) accounts for entropy production due to viscous heating in a weakly collisional magnetized plasma (see, e.g., Hollweg 1985). These equations have been considered in previous works investigating the dynamics of the weakly collisional ICM with a single ion species, i.e., in the case where the concentration of the other ion species is $c=0$, see, e.g., Kunz (2011); Parrish et al. (2012); Kunz et al. (2012), and references therein.

Equations (1)-(5) describe the dynamics of a dilute binary

\footnotetext{
1 This analysis can be generalized to consider $N$ species.

${ }^{2}$ For the sake of simplicity, we do not consider here the effects of thermodiffusion, baro-diffusion, etc. (Landau \& Lifshitz 1959).
}

mixture in the low-collisionality regime and they differ from standard MHD in three important respects.

(i) In a weakly collisional magnetized plasma the pressure tensor $\mathrm{P} \equiv p_{\perp} \mathbf{I}+\left(p_{\|}-p_{\perp}\right) \hat{\boldsymbol{b}} \hat{\boldsymbol{b}}$ is anisotropic. If the frequency of ion collisions $\nu_{i i}$ in single ion species magnetofluid is large compared to the rate of change $d / d t$ of all the fields involved, then the anisotropic part of the pressure tensor is small compared to its isotropic part $P \equiv 2 p_{\perp} / 3+p_{\|} / 3$ and (see, e.g., Hollweg 1985; Schekochihin et al. 2005)

$$
\frac{\left|p_{\|}-p_{\perp}\right|}{P}=\frac{3}{\nu_{i i}}\left|\frac{d}{d t}\left(\ln B \rho^{-2 / 3}\right)\right| \ll 1 .
$$

The anisotropic component of the pressure tensor in the momentum equation gives rise to the phenomenon known as Braginskii viscosity. For small pressure anisotropy ${ }^{3}$ this contribution is usually written as

$$
p_{\|}-p_{\perp}=3 \eta_{0}\left(\hat{\boldsymbol{b}} \hat{\boldsymbol{b}}-\frac{1}{3} \boldsymbol{l}\right): \nabla \boldsymbol{v},
$$

where $\eta_{0}$ is the largest of the coefficients in the viscous stress tensor derived by Braginskii (1965). In order to account for the effects of collisions between ions of different species in the binary mixture, we replace the $\nu_{i i}$ by an effective ion-ion collision frequency $\nu_{i i}^{\text {eff }}$, which we define in Appendix A.

(ii) Heat flows mainly along magnetic field lines, because the electron mean free path is large compared to its Larmor radius. This process is modeled by the second term on the right-hand side of Equation (4) via

$$
\boldsymbol{Q}_{\mathrm{s}} \equiv-\chi \underline{(\cdot \nabla) T} \text {, }
$$

where $T$ is the plasma temperature, assumed to be the same for ions and electrons, and $\chi$ is the thermal conductivity predominately due to electrons (Spitzer 1962; Braginskii 1965),

$$
\chi \approx 6 \times 10^{-7} T^{5 / 2} \mathrm{erg} \mathrm{cm}^{-1} \mathrm{~s}^{-1} \mathrm{~K}^{-1} .
$$

(iii) The composition of fluid elements can change due to particle fluxes. Considering the flux of particles

$$
\left.\boldsymbol{Q}_{\mathrm{c}} \equiv-D \underline{D} \cdot \boldsymbol{\nabla}\right) c \text {, }
$$

on the right-hand side of Equation (5) ensures that the diffusion of ions is mainly along magnetic field lines. This is a good approximation when the plasma is dilute enough for the ion mean free path to be large compared to the ion Larmor radius. The concentration $c$ is related to the mean molecular weight $\mu$ via

$$
\frac{1}{\mu} \equiv(1-c) \frac{\left(1+Z_{1}\right)}{\mu_{1}}+c \frac{\left(1+Z_{2}\right)}{\mu_{2}},
$$

where $\mu_{i}$ and $Z_{i}$, with $i=1,2$, are the molecular weights and the atomic numbers for the two ion species. The isotropic part of the pressure tensor is thus

$$
P=\frac{\rho k_{\mathrm{B}} T}{\mu m_{\mathrm{H}}},
$$

where $k_{\mathrm{B}}$ is the Boltzmann constant and $m_{\mathrm{H}}$ is the atomic mass unit.

3 In principle, appropriate closure approximations, see, e.g., Snyder et al. (1997) may be adopted to address the high-collisionality regime starting from the Chew-Goldberger-Low, or CGL, limit (Chew et al. 1956). A detailed application of this approach can be found in the context of accretion disks in Sharma et al. (2003). 


\subsection{Initial Background State}

We consider a weakly magnetized, plane-parallel atmosphere in a constant gravitational field $\boldsymbol{g} \equiv-g \hat{\boldsymbol{z}}$. The background magnetic field is weak enough that the mechanical equilibrium of the atmosphere, with scaleheight $H$, is maintained via $d P / d z=-g \rho$. We assume that the medium is stratified in density, temperature, and composition along the vertical $z$-direction. In the equilibrium state, all the particles in the plasma are assumed to be described by a Maxwellian distribution with the same temperature, so that $p_{\|} \equiv p_{\perp}$ initially.

In general, the background heat and particle fluxes do not vanish, i.e., $\hat{\boldsymbol{b}} \cdot \boldsymbol{\nabla} T \neq 0$ and $\hat{\boldsymbol{b}} \cdot \boldsymbol{\nabla} c \neq 0$, unless the magnetic field and the background gradients are orthogonal. The existence of a well-defined steady state, i.e., $\boldsymbol{\nabla} \cdot \boldsymbol{Q}_{\mathrm{s}}=\boldsymbol{\nabla} \cdot \boldsymbol{Q}_{\mathrm{c}}=0$, demands that the background fluxes should be linear functions of the distance along the direction of the magnetic field. However, even if this condition is not strictly satisfied, the dynamics of the modes that we consider is unlikely to be significantly affected if the local dynamical timescale is short compared to the timescale in which the entire system evolves (see also Quataert 2008).

\subsection{Validity of the Braginskii-MHD Approximation}

If the pressure anisotropy grows beyond $\left|p_{\|}-p_{\perp}\right| / P \simeq$ $\beta^{-1}$, where the plasma $\beta \equiv v_{\mathrm{th}}^{2} / v_{\mathrm{A}}^{2}, v_{\mathrm{th}} \equiv(2 P / \rho)^{1 / 2}$ is the thermal speed, and $v_{\mathrm{A}} \equiv B /(4 \pi \rho)^{1 / 2}$ is the Alfvén speed, the Braginskii-MHD approximation embodied in Equations (11) (4) becomes ill-posed. This is because the viscous term introduced in Equation (7) not only fails to damp all the kinetic energy available at the viscous scale but also triggers various fast-growing, micro-scale plasma instabilities, such as mirror and firehose (see Schekochihin et al. 2005, 2008 and references therein). The growth rates of these instabilities are of the order of $\gamma \simeq k_{\|} v_{\text {th }}\left|p_{\|}-p_{\perp}\right| / P$ and thus they can dominate the plasma dynamics at very small scales if $\left|p_{\|}-p_{\perp}\right| / P \gtrsim \beta^{-1}$. This poses a challenge in numerical simulations addressing the non-linear dynamics of the BraginskiiMHD equations since these instabilities grow formally at the grid scale and some procedure must be devised in order to capture their effects (see Kunz et al. 2012 for a detailed discussion).

One possibility, which would prevent the micro-instabilities from operating at once, is to ignore the effects of pressure anisotropies and the associated Braginskii viscosity. This was the approach followed in the seminal papers on the MTI (Balbus 2001) and the HBI (Quataert 2008), which showed that both instabilities grow on the dynamical timescale set by $\omega_{\text {dyn }}^{-1} \simeq(H / g)^{1 / 2}$. However, because the timescales involved in viscous processes are only a factor of a few longer than the dynamical timescales on which both the HBI and the MTI operate, accounting for small pressure anisotropies can affect the range of wavenumber over which these instabilities operate, as well as their growth rates (Kunz 2011). Furthermore, the timescales involved in processes related to ion-diffusion are only a factor of a few larger than those involved in viscous processes (see below). Since our aim is to understand the interplay of the various processes involved in determining the stability of a medium stratified in both temperature and composition, we retain the term accounting for Braginskii viscosity, Equation (7), in the momentum Equation (2). We argue next that Equations (11)-(4) provide an adequate framework to analyze the dynamics of small amplitude perturbations of the stratified atmosphere described.

The equilibrium background state over which we perform the stability analysis is such that $p_{\|} \equiv p_{\perp}$, and thus there is an initial period of time for which the pressure anisotropy will remain small enough that these plasma-micro instabilities can be ignored. Kunz et al. (2012) estimate that the amplitude to which the fluctuations in the magnetic field can grow before these instabilities set in, and thus Equations (11)-(4) remain self-consistent, is roughly given by $\delta B_{\|} / B \simeq H /\left(\beta \lambda_{\mathrm{mfp}}\right)$, where $\lambda_{\mathrm{mfp}}$ stands for the mean free path between particle collisions. We can estimate this value for the ICM as follows. The plasma $\beta$ increases from $\simeq 10^{2}$ in the inner cluster regions to $\simeq 10^{4}$ in the outer parts, while the ratio $H / \lambda_{\mathrm{mfp}}$ decreases from $10^{3}-10^{2}$ in the cluster core to $10^{2}-10$ in the outer region. Therefore, the ratio $H /\left(\beta \lambda_{\mathrm{mfp}}\right)$ is larger than unity in the central regions of a typical galaxy cluster and decreases outward to roughly $10^{-2}$. We thus conclude that, for the sake of performing a linear mode analysis, which is only formally valid when the fluctuations of all the physical variables are small, e.g., $\delta B / B \ll 1$, the Braginskii-MHD Equations (11)-(4) describes the problem under consideration self-consistently. These micro-instabilities are likely to play an important role in the subsequent non-linear dynamics, but addressing this regime is beyond the scope of this study.

\section{STABILITY ANALYSIS}

\subsection{Linearized Equations}

The modes of interest have associated timescales that are long compared to the sound crossing time and it thus suffices to work in the Boussinesq approximation (Balbus 2001; Quataert 2008). In this limit, the equations for the linear perturbations $\delta \simeq e^{\sigma t+i \boldsymbol{k} \cdot \boldsymbol{x}}$ become开

$$
\begin{aligned}
\sigma \delta \boldsymbol{v}= & -g \frac{\delta \rho}{\rho} \hat{\boldsymbol{z}}-i \boldsymbol{k} v_{\mathrm{th}}^{2}\left(\frac{\delta p_{\perp}}{P}+\frac{1}{\beta} \frac{\delta B_{\|}}{B}\right)+i k_{\|} v_{\mathrm{A}}^{2} \frac{\delta \boldsymbol{B}}{B} \\
& -\underline{3} k_{\|}^{2} \nu_{\|} \delta v_{\|} \\
\sigma \delta \boldsymbol{B}= & i k_{\|} B \delta \boldsymbol{v} \\
\sigma \frac{\delta \rho}{\rho}= & \frac{N^{2}}{g} \delta v_{z}+\frac{\gamma-1}{\gamma} \kappa k_{\|}^{2} \frac{\delta T}{T} \\
& -i \frac{\gamma-1}{\gamma} \kappa \boldsymbol{k} \cdot\left(\frac{d \ln T}{d z} \delta b_{z} \pm b_{z} \frac{d \ln T}{d z} \frac{\delta \boldsymbol{B}_{\perp}}{B}\right) \\
\sigma \frac{\delta \mu}{\mu}= & -\frac{d \ln \mu}{d z} \delta v_{z}-D k_{\|}^{2} \frac{\delta \mu}{\mu} \\
& +i D \boldsymbol{k} \cdot\left(\frac{d \ln \mu}{d z} \delta b_{z} \pm b_{z} \frac{d \ln \mu}{d z} \frac{\delta \boldsymbol{B}_{\perp}}{B}\right)
\end{aligned}
$$

Here, we have defined the anisotropic viscosity coefficient

$$
\nu_{\|}=\frac{1}{2} \frac{v_{\mathrm{th}}^{2}}{\nu_{i i}^{\mathrm{eff}}},
$$

where $\nu_{i i}^{\text {eff }}$ is an effective collision rate for the binary mixture (see Appendix $\overline{\mathrm{A}}$ ), and the thermal diffusion coefficient,

$$
\kappa \equiv \frac{\chi T}{P} .
$$

\footnotetext{
${ }^{4}$ The effects of Braginskii viscosity in the thermal evolution of the plasma, which appear in Equation (4) as proportional to $\left(p_{\|}-p_{\perp}\right)^{2}$, are of higher order and thus they do not contribute to the linear analysis.
} 
We have also introduced the Brunt-Väisälä frequency, $N^{2}$, which, in a medium stratified in density, temperature, and composition, is given by

$$
N^{2} \equiv \frac{g}{\gamma} \frac{d}{d z} \ln P \rho^{-\gamma}=g \frac{d}{d z} \ln \left(\frac{P^{\frac{1-\gamma}{\gamma}} T}{\mu}\right) .
$$

Note that, in agreement with the Boussinesq approximation, the velocity perturbations satisfy $\boldsymbol{k} \cdot \delta \boldsymbol{v}=0$ and the fluctuations in density, temperature, and mean molecular weight are related via

$$
\frac{\delta \rho}{\rho}+\frac{\delta T}{T}-\frac{\delta \mu}{\mu}=0
$$

\subsection{Relevant Timescales Across a Mode}

Because of the several physical processes that play a role in the stability of the dilute atmosphere, the dispersion relation corresponding to Equations (13)- 16) is rather involved. It is thus useful to understand the hierarchy of the timescales involved in the dynamics of a single Fourier mode in order to make sensible approximations. The analysis below applies to the range of local modes with wavevectors parallel to the magnetic field for which the fluid approach is valid, i.e., $H^{-1}<k_{\|}<\lambda_{\mathrm{mfp}}^{-1}$, or

$$
\sqrt{K_{\mathrm{n}}}<k_{\|} \sqrt{\lambda_{\mathrm{mfp}} H}<\sqrt{K_{\mathrm{n}}^{-1}},
$$

where we have defined the Knudsen number

$$
K_{\mathrm{n}} \equiv \frac{\lambda_{\mathrm{mfp}}}{H} .
$$

The inverse timescales characterizing the diffusion of heat, momentum, and particles along magnetic field lines are

$$
\begin{aligned}
\tau_{\mathrm{c}}^{-1} & \equiv(\boldsymbol{k} \cdot \hat{\boldsymbol{b}})^{2} \kappa \frac{(\gamma-1)}{\gamma}, \\
\tau_{\mathrm{v}}^{-1} & \equiv(\boldsymbol{k} \cdot \hat{\boldsymbol{b}})^{2} 3 \nu_{\|}, \\
\tau_{\mathrm{d}}^{-1} & \equiv(\boldsymbol{k} \cdot \hat{\boldsymbol{b}})^{2} D .
\end{aligned}
$$

For a given mode, the ratio between these timescales is independent of the direction of the wavevector characterizing the perturbation and the background magnetic field and is set by plasma processes. Because heat conduction is mostly due to electrons, while viscous processes are dominated by the dynamics of ions, it could be expected that the associated timescales would satisfy $\tau_{\mathrm{c}}^{-1} \gg \tau_{\mathrm{d}}^{-1}$. However, this is not the case and a simple estimate leads to $\tau_{\mathrm{c}}^{-1} \simeq 6 \tau_{\mathrm{v}}^{-1}$ (Kunz 2011). It could be argued that the timescales involved in viscous and diffusion processes should be of the same order because it is mainly the ion dynamics what determines both of them. A detailed analysis of the diffusion coefficient for a binary mixture of ions (see Appendix $\mathrm{A}$ ) shows that $\tau_{\mathrm{d}}^{-1} \simeq 9 \tau_{\mathrm{v}}^{-1}$ for primordial composition ( $c \simeq 0.25$ or $\mu \simeq 0.6$ ) and decreases toward $\tau_{\mathrm{d}}^{-1} \simeq 3 \tau_{\mathrm{v}}^{-1}$ for the compositions expected at the inner core of galaxy clusters according to recent models for helium sedimentation (Bulbul et al. 2011). Since we will be mostly concerned with the two regimes $\tau_{\mathrm{c}}^{-1} \gg \omega_{\text {dyn }}$ or $\omega_{\text {dyn }} \gg \tau_{\mathrm{c}}^{-1}$, as long as the ratio $\tau_{\mathrm{d}}^{-1} / \tau_{\mathrm{v}}^{-1}$ is not too small, its particular value will not affect our main conclusions, and we will thus consider that $\tau_{\mathrm{d}}^{-1} \simeq \tau_{\mathrm{v}}^{-1}$.

On the other hand, whether the timescales set by plasma processes are fast or slow compared to the dynamical timescale $\omega_{\mathrm{dyn}}^{-1} \equiv(H / g)^{1 / 2}$ depends not only on the wavelength of the mode but also on the direction of the wavevector characterizing the perturbation with respect to the background magnetic field. In particular, as shown in (Kunz 2011), the timescale characterizing conduction across a mode with parallel wavenumber $k_{\|}$is related to the dynamical timescale via

$$
\tau_{\mathrm{c}}^{-1} \simeq 10 k_{\|}^{2} \lambda_{\mathrm{mfp}} H \omega_{\mathrm{dyn}},
$$

where we have assumed $\gamma=5 / 3$ in Equation (23). Thus conduction is faster than the dynamical time, i.e., $\tau_{\mathrm{c}}^{-1} / \omega_{\text {dyn }}>1$, if $k_{\|}\left(\lambda_{\mathrm{mfp}} H\right)^{1 / 2}>1 / 3$. If the wavelength of the mode is shorter than this by a factor of $\tau_{\mathrm{c}}^{-1} / \tau_{\mathrm{v}}^{-1} \simeq 6$, e.g., $k_{\|}\left(\lambda_{\mathrm{mfp}} H\right)^{1 / 2} \gg 1$, then viscous and diffusive processes are also faster than the dynamical timescale. Therefore, as a useful approximate criterion, whether $k_{\|}\left(\lambda_{\mathrm{mfp}} H\right)^{1 / 2}$ is much larger or smaller than unity defines whether the timescales associated with plasma processes, for that given mode, are shorter or longer than the dynamical time. We will thus consider two different regimes which we refer to as the "fast" and "slow" conduction limit, where the timescales associated with the modes considered satisfy, respectively,

$$
\begin{aligned}
& \tau_{\mathrm{c}}^{-1}>\tau_{\mathrm{v}}^{-1} \simeq \tau_{\mathrm{d}}^{-1} \gg \omega_{\mathrm{dyn}} \quad \text { if } \quad k_{\|} \gg\left(\lambda_{\mathrm{mfp}} H\right)^{-1 / 2}, \\
& \omega_{\mathrm{dyn}} \gg \tau_{\mathrm{c}}^{-1}>\tau_{\mathrm{v}}^{-1} \simeq \tau_{\mathrm{d}}^{-1} \quad \text { if } \quad k_{\|} \ll\left(\lambda_{\mathrm{mfp}} H\right)^{-1 / 2} .
\end{aligned}
$$

\subsection{The Weak Magnetic Field Limit}

All the timescales related to plasma processes discussed above depend only on the direction of a given wavevector with respect to the magnetic field. The only time scale that depends explicitly on the strength of the field is the one associated with the Alfvén frequency $\omega_{\mathrm{A}} \equiv \boldsymbol{k} \cdot \boldsymbol{v}_{\mathrm{A}}$. In order to keep the problem tractable, and given that we are already dealing with four different timescales, we will focus on the case where the magnetic field is so weak that its only physical role is to channel the flux of heat and ions. The advantage of this limit is that it allows us to address the anisotropic dynamics of the weakly collisional magnetized medium without introducing explicitly the timescale associated with $\omega_{\mathrm{A}}$.

In what follows we focus our attention on modes for which magnetic tension is unimportant and thus $\omega_{\mathrm{A}} \ll$ $\min \left\{\tau_{\mathrm{c}}^{-1}, \omega_{\mathrm{dyn}}\right\}$. This approximation will be valid for two different ranges of parallel wavenumbers depending on whether $k_{\|}\left(\lambda_{\mathrm{mfp}} H\right)^{1 / 2}$ is much larger or smaller than unity. For the modes for which conduction is faster than the dynamical timescale, i.e., $k_{\|}\left(\lambda_{\mathrm{mfp}} H\right)^{1 / 2} \gg 1$, we must require $\omega_{\mathrm{A}} \ll \omega_{\text {dyn }} \ll \tau_{\mathrm{c}}^{-1}$. Using the definitions $v_{\text {th }}=(g H)^{1 / 2}$, $\beta=v_{\mathrm{th}}^{2} / v_{\mathrm{A}}^{2}$, and $K_{n}=\lambda_{\mathrm{mfp}} / H$, we obtain that magnetic tension is negligible provided that

$$
1 \ll k_{\|} \sqrt{\lambda_{\mathrm{mfp}} H} \ll \sqrt{\beta K_{n}},
$$

and thus

$$
\omega_{\mathrm{A}} \simeq 0 \text { for } \tau_{\mathrm{c}}^{-1} \gg \omega_{\mathrm{dyn}} \text { if } \beta K_{\mathrm{n}} \gg 1 .
$$

For the modes for which conduction is slow compared to the dynamical timescale, i.e., $k_{\|}\left(\lambda_{\mathrm{mfp}} H\right)^{1 / 2} \ll 1$, we must require $\omega_{\mathrm{A}} \ll \tau_{\mathrm{c}}^{-1} \ll \omega_{\mathrm{dyn}}$. This is satisfied if

$$
\frac{1}{10} \frac{1}{\sqrt{\beta K_{n}}} \ll k_{\|} \sqrt{\lambda_{\mathrm{mfp}} H} \ll 1,
$$


and therefore

$$
\omega_{\mathrm{A}} \simeq 0 \text { for } \omega_{\mathrm{dyn}} \gg \tau_{\mathrm{c}}^{-1} \text { if } \beta K_{\mathrm{n}} \gg 10^{-2} .
$$

The plasma $\beta$ ranges from $10^{4}$ in the outskirts of the ICM down to $10^{2}$ in the centers of cool core clusters (Carilli \& Taylor 2002), and the product $\beta K_{\mathrm{n}}$ ranges from $10^{3}$ in the outskirts of the ICM decreasing to $10^{-1}$ in the inner regions. Thus the effects of magnetic tension can be important in the inner cluster regions. We address the implications of neglecting magnetic tension when analyzing the stability of the ICM in further detail in Section 7

\subsection{General Dispersion Relation for the Dilute, Weakly Magnetized Medium}

The dispersion relation corresponding to the set of equations for the linear perturbations 13 -16 is

$$
\sum_{i=0}^{4} A_{i} \sigma^{5-i}+\tau_{\mathrm{v}}^{-1} \sum_{i=1}^{5} B_{i} \sigma^{5-i}=0,
$$

where the coefficients $A_{i}$ are given by

$$
\begin{aligned}
A_{0} & \equiv 1, \\
A_{1} & \equiv \tau_{\mathrm{c}}^{-1}, \\
A_{2} & \equiv \frac{\left(k_{x}^{2}+k_{y}^{2}\right)}{k^{2}} N^{2}+\tau_{\mathrm{d}}^{-1} \tau_{\mathrm{c}}^{-1}, \\
A_{3} & \equiv \tau_{\mathrm{c}}^{-1} g\left\{\frac{d \ln T}{d z} \frac{\mathcal{K}}{k^{2}}-\frac{d \ln \mu}{d z} \frac{\left(k_{x}^{2}+k_{y}^{2}\right)}{k^{2}}\right\} \\
& +\tau_{\mathrm{d}}^{-1} \frac{\left(k_{x}^{2}+k_{y}^{2}\right)}{k^{2}} N^{2}, \\
A_{4} & \equiv \tau_{\mathrm{d}}^{-1} \tau_{\mathrm{c}}^{-1} N_{T / \mu}^{2} \frac{\mathcal{K}}{k^{2}},
\end{aligned}
$$

while the $B_{i}$ read

$$
\begin{aligned}
& B_{1} \equiv \frac{k_{\perp}^{2}}{k^{2}} \\
& B_{2} \equiv \tau_{\mathrm{c}}^{-1} \frac{k_{\perp}^{2}}{k^{2}}, \\
& B_{3} \equiv \tau_{\mathrm{d}}^{-1} \tau_{\mathrm{c}}^{-1} \frac{k_{\perp}^{2}}{k^{2}}+N^{2} \frac{b_{x}^{2} k_{y}^{2}}{k^{2}}, \\
& B_{4} \equiv\left(\tau_{\mathrm{c}}^{-1} N_{T / \mu}^{2}+\tau_{\mathrm{d}}^{-1} N^{2}\right) \frac{b_{x}^{2} k_{y}^{2}}{k^{2}}, \\
& B_{5} \equiv \tau_{\mathrm{d}}^{-1} \tau_{\mathrm{c}}^{-1} N_{T / \mu}^{2} \frac{b_{x}^{2} k_{y}^{2}}{k^{2}} .
\end{aligned}
$$

Here we have defined

$$
\begin{aligned}
\mathcal{K} & \equiv\left(1-2 b_{z}^{2}\right)\left(k_{x}^{2}+k_{y}^{2}\right)+2 b_{x} b_{z} k_{x} k_{z}, \\
& =b_{x}^{2} k^{2}-k_{\perp}^{2}+b_{x}^{2} k_{y}^{2}, \\
& =-b_{z}^{2} k^{2}+k_{\|}^{2}+b_{x}^{2} k_{y}^{2},
\end{aligned}
$$

and the two quantities

$$
\begin{aligned}
N_{T \mu}^{2} & \equiv g \frac{d}{d z} \ln (T \mu), \\
N_{T / \mu}^{2} & \equiv g \frac{d}{d z} \ln \left(\frac{T}{\mu}\right),
\end{aligned}
$$

which appear naturally when thermal and composition gradients are considered. The dispersion relation (33) is identical to the one derived in (Kunz 2011) in the limit in which $\omega_{\mathrm{A}}$, $d \mu / d z$, and $D$ vanish. Note that in the limit of a constant composition gradient, i.e., $d \mu / d z \rightarrow 0$, both $N_{T \mu}^{2}$ and $N_{T / \mu}^{2}$ $\rightarrow g d \ln T / d z$, which is the logarithmic gradient that plays an important role in the stability of a homogeneous, dilute, weakly magnetized medium.

\section{THE FAST CONDUCTION LIMIT}

We first consider the stability of the modes for which conduction is faster than the dynamical time, i.e., $\tau_{\mathrm{c}}^{-1} \gg \omega_{\text {dyn }}$. This is the regime that corresponds to the well-studied HBI and MTI.

\subsection{Limit of No Ion-Diffusion}

As a first step toward understanding the effects of composition gradients in the behavior of the HBI and the MTI, we neglect the diffusion of ions along magnetic field lines by setting $D=0$. Because we are considering the timescales for ion-diffusion and viscous processes to be of the same order, i.e., $\tau_{\mathrm{v}}^{-1} \simeq \tau_{\mathrm{d}}^{-1}$, we also ignore here the effects of viscosity and set $\nu_{\|}=0$ for consistency. For the modes for which $\tau_{\mathrm{c}}^{-1} \gg \omega_{\mathrm{dyn}}$, the dispersion relation (33) yields a (fast) decaying solution, $\sigma \approx-\tau_{\mathrm{c}}^{-1}$, together with the two slow modes

$$
\sigma^{2} \approx-g\left\{\frac{d \ln T}{d z} \frac{\mathcal{K}}{k^{2}}-\frac{d \ln \mu}{d z} \frac{\left(k_{x}^{2}+k_{y}^{2}\right)}{k^{2}}\right\} .
$$

In a homogeneous plasma, these slow modes contain the wellknown HBI and MTI, depending on the direction of the background magnetic field, i.e.,

$$
\begin{array}{ll}
\sigma_{\mathrm{HBI}}^{2} \approx g \frac{d \ln T}{d z} \frac{k_{\perp}^{2}}{k^{2}} & \text { for } b_{z}=1, \\
\sigma_{\mathrm{MTI}}^{2} \approx-g \frac{d \ln T}{d z} \frac{k_{x}^{2}+k_{y}^{2}}{k^{2}} & \text { for } b_{x}=1 .
\end{array}
$$

The conditions for the excitation of the HBI and the MTI are thus

$$
\begin{aligned}
& \frac{d \ln T}{d z}>0 \quad \text { HBI-unstable } \\
& \frac{d \ln T}{d z}<0 \quad \text { MTI-unstable. }
\end{aligned}
$$

Note that in both cases, the fastest growing modes are those with wavevectors perpendicular to the gravitational field.

\subsubsection{Heat- and Particle-Flux Driven Buoyant Instability

$$
(D=0)
$$

Consider a magnetic field parallel to the gravitational field, i.e., $b_{z}=1$, and thus $k_{x}^{2}+k_{y}^{2}=k_{\perp}^{2}$. The modified version of the modes that become HBI-unstable in a homogeneous medium is given by Equation 49]. In the medium stratified in composition, these modes become

$$
\sigma^{2} \approx g \frac{d \ln (T \mu)}{d z} \frac{k_{\perp}^{2}}{k^{2}} .
$$

Therefore, neglecting viscous and diffusion processes in a medium which is stratified in the mean molecular weight 
(a)

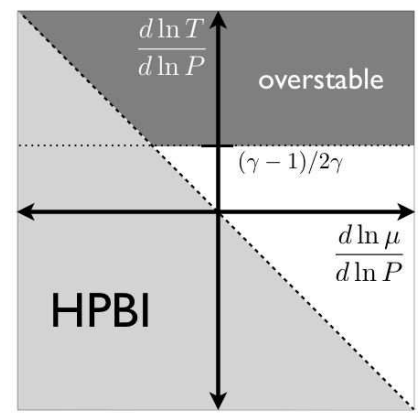

$B=B_{z} ; D=0$ (b) Diffusion driven modes

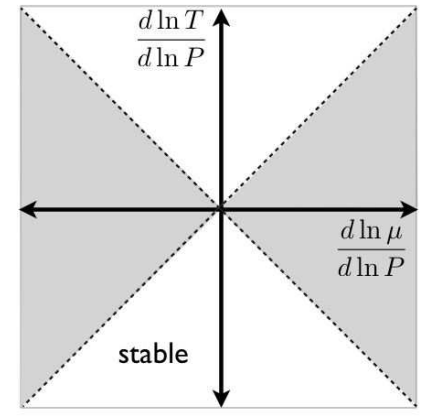

$B=B_{z} ; D \neq 0$ (c)

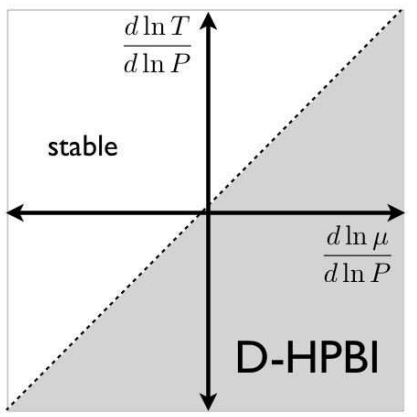

$B=B_{z} ; D \neq 0$ (d)

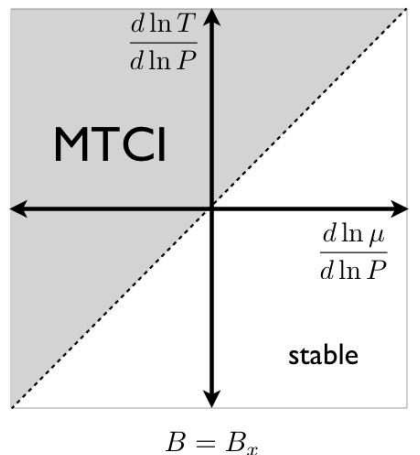

Figure 2. Graphic representation of the stability of modes for which conduction is fast compared to the dynamical timescale, i.e., $\tau_{\mathrm{c}}^{-1} \gg \omega_{\mathrm{dyn}}$. The various panels show the unstable regions (gray) for each of the modes that can be excited when the background magnetic field is parallel $(a, b$, and $c$ ) or perpendicular (d) to the background thermal and composition gradients. The horizontal dotted line in panel (a) represents $d \ln T / d \ln P=(\gamma-1) / 2 \gamma$; and the dashed lines correspond $d \ln T / d \ln P= \pm d \ln \mu / d \ln P$. Panel (a) shows the region of parameter space which is unstable to the heat- and particle-flux-driven buoyancy instability (HPBI), together with the regions that are overstable to gravity modes. If ions can diffuse efficiently along magnetic field lines, i.e., $D \neq 0$, there are unstable modes that can be driven by diffusion, whether $\omega_{\mathrm{dyn}}>\tau_{\mathrm{d}}^{-1} \simeq \tau_{\mathrm{v}}^{-1}$ (b) or $\tau_{\mathrm{d}}^{-1} \simeq \tau_{\mathrm{v}}^{-1}>\omega_{\mathrm{dyn}}$ (c). Panel (d) shows the region that is unstable to the magneto-thermo-compositional instability (MTCI); in this case, the criterion for instability is insensitive to the value of the diffusion coefficient $D$.

leads to modes that are unstable to a Heat- and Particle-fluxdriven Buoyant Instability (HPBI) if

$$
\frac{d \ln T}{d z}>-\frac{d \ln \mu}{d z} \quad \text { HPBI-unstable }
$$

The threshold temperature gradient for instability can be negative if the mean molecular weight increases with height.

The combination of temperature and composition gradients that is HPBI-unstable is shown in panel (a) of Figure 2 where, for the sake of convenience, we have defined dimensionless variables $(d \ln \mu / d \ln P, d \ln T / d \ln P)$ in terms of the logarithmic pressure gradient $d \ln P / d z \equiv-1 / H$.

\subsubsection{Overstable Modes $(D=0)$}

Balbus \& Reynolds (2010) showed that temperature gradients that are stable to the HBI can nevertheless be subject to overstable gravity modes. This result can be extended to include non-vanishing composition gradients, i.e., there is a range of modes with $\tau_{\mathrm{c}}^{-1}>\omega_{\text {dyn }}$ and $\sigma \simeq \omega_{\text {dyn }}$ that can become overstable when the heat- and particle-flux-driven buoyancy instability (HPBI) does not operate. Calculating these modes requires retaining higher order terms in the dispersion relation, which becomes

$$
\sigma^{3}+\tau_{\mathrm{c}}^{-1} \sigma^{2}+\frac{k_{\perp}^{2}}{k^{2}} N^{2} \sigma-\tau_{\mathrm{c}}^{-1} N_{T \mu}^{2} \frac{k_{\perp}^{2}}{k^{2}}=0 .
$$

In this regime, we can treat the first and third terms on the left-hand side as perturbations and extend the solutions (54) to contain corrections of order $\omega_{\mathrm{dyn}}^{2} / \tau_{\mathrm{c}}^{-1}$ :

$$
\sigma \approx \pm i \sqrt{-N_{T \mu}^{2} \frac{k_{\perp}^{2}}{k^{2}}}-\frac{N^{2}+N_{T \mu}^{2}}{2 \tau_{\mathrm{c}}^{-1}} .
$$

Therefore, modes that are stable according to the HPBIstability criterion, i.e., $N_{T \mu}^{2}<0$ (Equation [55]), can become overstable if $N_{T \mu}^{2}<-N^{2}$. In terms of the dimensionless variables introduced earlier, these requirements become

$$
\frac{d \ln T}{d \ln P}>\max \left\{-\frac{d \ln \mu}{d \ln P}, \frac{\gamma-1}{2 \gamma}\right\}
$$

HPBI-overstable .(58)
The combination of temperature and composition gradients that is subject to overstability is shown in dark gray in panel (a) of Figure 2

\subsubsection{Magneto-Thermo-Compositional Instability $(D=0)$}

In order to understand how the MTI is modified in the presence of composition gradients we consider a horizontal magnetic field along the $x$-axis, i.e., $b_{x}=1$ and focus on the modes for which $\tau_{\mathrm{c}}^{-1} \gg \omega_{\text {dyn. }}$. In the presence of a gradient in the mean molecular weight, Equation (49) gives the generalization of the modes that become MTI-unstable

$$
\sigma^{2} \approx-g \frac{d \ln (T / \mu)}{d z} \frac{k_{x}^{2}+k_{y}^{2}}{k^{2}} .
$$

Thus a non-vanishing gradient in the mean molecular weight sets an upper bound for the temperature gradients that are magneto-thermo-compositional instability (MTCI)-unstable

$$
\frac{d \ln T}{d z}<\frac{d \ln \mu}{d z} \quad \text { MTCI-unstable. }
$$

Panel (d) in Figure 2 shows a graphical representation of the region of parameter space that is subject to unstable MTCI modes in a medium that is stratified in composition and temperature.

\subsection{Ion-Diffusion Along Magnetic Field Lines}

We now analyze the effects of including ion-diffusion induced by the background composition gradients. Since, for a given mode, viscous and diffusion timescales are of the same order, i.e., $\tau_{\mathrm{y}}^{-1} \simeq \tau_{\mathrm{d}}^{-1}$, we also consider the effects of anisotropic viscosity for consistency.

\subsubsection{Heat-and Particle-Flux-Driven Buoyant Instability

$$
(D \neq 0)
$$

Starting from the general dispersion relation in the case where $b_{z}=1$, it can be seen that, if we consider modes for which conduction is faster than any other timescale, there is a fast decaying solution $\sigma=-\tau_{\mathrm{c}}^{-1}$ which can be used to self- 
consistently obtain three more modes satisfying

$$
\begin{aligned}
\sigma^{3}+\left(\tau_{\mathrm{d}}^{-1}+\tau_{\mathrm{v}}^{-1} \frac{k_{\perp}^{2}}{k^{2}}\right) \sigma^{2} & +\frac{k_{\perp}^{2}}{k^{2}}\left(\tau_{\mathrm{d}}^{-1} \tau_{\mathrm{v}}^{-1}-N_{T \mu}^{2}\right) \sigma \\
& -\tau_{\mathrm{d}}^{-1} N_{T / \mu}^{2} \frac{k_{\perp}^{2}}{k^{2}}=0
\end{aligned}
$$

However, the leading order solutions to this dispersion relation depend on whether the dynamical timescale is fast or slow with respect to the diffusion and viscous timescales across the mode. We thus need to consider these two cases separately.

Slow diffusion. The modes for which $\omega_{\mathrm{dyn}}>\tau_{\mathrm{v}}^{-1} \simeq \tau_{\mathrm{d}}^{-1}$ contain the generalization of the HBI for non-vanishing composition gradients

$$
\sigma^{2} \approx N_{T \mu}^{2} \frac{k_{\perp}^{2}}{k^{2}}
$$

which grows dynamically if $N_{T \mu}^{2}>0$, or $d T / d z>-d \mu / d z$. This is the same condition for the onset of the HPBI in Equation (55) in the absence of diffusion. It is worth mentioning that even if the combination of temperature and composition gradients is such that $N_{T \mu}^{2}<0$ (and thus the plasma is HPBI-stable), overstable modes can be excited just as in the non-diffusive case. In fact, the condition for overstability can be shown to be exactly similar to (58), provided that $\omega_{\text {dyn }}>\max \left\{\tau_{\mathrm{c}}^{-1} \tau_{\mathrm{v}}^{-1}, \tau_{\mathrm{c}}^{-1} \tau_{\mathrm{d}}^{-1}\right\}$. There also exists a slower mode driven by ion-diffusion

$$
\sigma \approx-\tau_{\mathrm{d}}^{-1} \frac{N_{T / \mu}^{2}}{N_{T \mu}^{2}},
$$

which grows in the region of parameter space where $N_{T / \mu}^{2} / N_{T \mu}^{2}<0$ or, in terms of the background gradients, wherever $d \ln T / d \ln P<|d \ln \mu / d \ln P|$, as it is shown in panel (b) of Figure 2. Note that these modes can grow on a diffusion timescale even if the system is HPBI stable, i.e., $N_{T \mu}^{2}<0$, provided that $N_{T / \mu}^{2}>0$. As we show below, this latter requirement becomes the deciding one for those modes for which diffusion is not slow compared to the dynamical time.

Fast diffusion. For the modes for which $\tau_{\mathrm{v}}^{-1} \simeq \tau_{\mathrm{d}}^{-1}>$ $\omega_{\text {dyn }}$, there are two solutions that decay on the diffusion and viscous timescales, i.e., $\sigma \approx-\tau_{\mathrm{d}}^{-1}$ and $\sigma \approx-\tau_{\mathrm{v}}^{-1} k_{\perp}^{2} / k^{2}$, and a third one

$$
\sigma \approx \frac{N_{T / \mu}^{2}}{\tau_{\mathrm{v}}^{-1}},
$$

which can become unstable if $N_{T / \mu}^{2}>0$. This region of parameter space in temperature and composition gradients is unstable to a diffusive version of the Heat- and Particle-fluxdriven Buoyant Instability (D-HPBI)

$$
\frac{d \ln T}{d z}>\frac{d \ln \mu}{d z} \quad \text { D-HPBI-unstable, }
$$

and it is shown in panel (c) of Figure 2

In summary, we conclude that when finite viscous and diffusion timescales are considered, there can be unstable modes

\footnotetext{
${ }^{5}$ When a finite diffusion timescale is considered, i.e., $D \neq 0$, the term that contributes to the generalization of the HBI is the second term inside brackets in the right hand side of Equation 16
}

driven by diffusion (with $\omega_{\mathrm{dyn}}>\tau_{\mathrm{d}}^{-1}$ ) even if the temperature and the composition gradients do not satisfy inequality (55), i.e., they are stable to the HPBI in the absence of diffusion, provided that $d \ln T>d \ln \mu$. Note that this is the very requirement for the existence of unstable modified HBI modes when finite diffusion timescales are relevant (with $\tau_{\mathrm{d}}^{-1}>\omega_{\mathrm{dyn}}$ ) and differs from the condition (55). We shall discuss the physical reason behind this change in condition of instability in Section 6 .

\subsubsection{Magneto-Thermo-Compositional Instability $(D \neq 0)$}

In order to understand how ion-diffusion driven by a composition gradient affects the MTI, we consider $D \neq 0$ and modes for which $\tau_{\mathrm{c}}^{-1} \gg \omega_{\text {dyn }}$ when $b_{x}=1$. In this case, the dispersion relation factorizes and leads to a fast decaying solution $\sigma=-\tau_{\mathrm{c}}^{-1}$ together with

$$
\sigma^{3}+\tau_{\mathrm{v}}^{-1} \frac{k_{\perp}^{2}}{k^{2}} \sigma^{2}+N_{T / \mu}^{2} \frac{k_{x}^{2}+k_{y}^{2}}{k^{2}} \sigma+\tau_{\mathrm{v}}^{-1} N_{T / \mu}^{2} \frac{k_{y}^{2}}{k^{2}}=0 .
$$

For the modes satisfying $\omega_{\text {dyn }}>\tau_{\mathrm{v}}^{-1}$, the three solutions to this cubic equation correspond to a decaying viscous mode

$$
\sigma \approx-\tau_{\mathrm{v}}^{-1} \frac{k_{y}^{2}}{k_{x}^{2}+k_{y}^{2}},
$$

and a pair of roots that contain the generalization of the MTI in the presence of a mean molecular weight gradient and $D \neq$ 0

$$
\sigma^{2} \approx-N_{T / \mu}^{2} \frac{k_{x}^{2}+k_{y}^{2}}{k^{2}}
$$

which grows dynamically if $N_{T / \mu}^{2}<0$, or $d T / d z>d \mu / d z$.

This is the same condition for the onset of the MTCI in Equation 60) in the absence of diffusion. As we found before, a non-vanishing gradient in the mean molecular weight sets a upper bound for the temperature gradients that are MTIunstable. This temperature gradient can be positive when the composition gradient is negative (i.e., mean molecular weight increasing with height). Note that this statement is independent of the value of the diffusion coefficient $D$, i.e., and thus of whether or not ions diffuse effectively along magnetic field lines on a dynamical timescale.

For the modes such that $\tau_{\mathrm{v}}^{-1}>\omega_{\mathrm{dyn}}$, the three solutions to Equation (66) correspond to another viscously damped mode

$$
\sigma \approx-\tau_{\mathrm{v}}^{-1} \frac{k_{\perp}^{2}}{k^{2}},
$$

together with a generalization of the modes identified as Alfvénic-MTI in Kunz (2011), i.e.,

$$
\sigma \approx-\frac{N_{T / \mu}^{2}}{2 \tau_{\mathrm{v}}^{-1}} \frac{k_{x}^{2}}{k_{\perp}^{2}} \pm i \sqrt{N_{T / \mu}^{2}} \frac{k_{y}}{k_{\perp}} .
$$

These modes grow dynamically if $N_{T / \mu}^{2}<0$, which corresponds again to the combination of temperature and composition gradients satisfying inequality (60).

\section{THE SLOW CONDUCTION LIMIT}

We now analyze the stability of the modes whose associated timescales satisfy $\omega_{\mathrm{dyn}} \gg \tau_{\mathrm{c}}^{-1}>\tau_{\mathrm{v}}^{-1} \simeq \tau_{\mathrm{d}}^{-1}$. For a homogeneous plasma, these modes encompass the overstable $g$-modes studied in Balbus \& Reynolds (2010). The set 
(a) Gravity modes

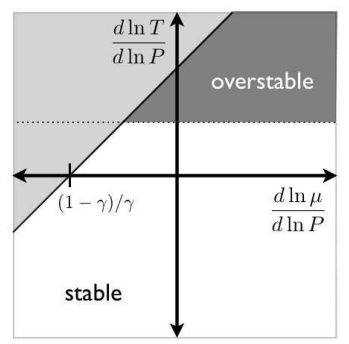

$B=B_{z} ; D=0$ (b) Conduction modes

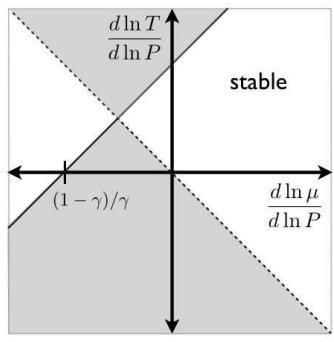

$B=B_{z} ; D=0$ (c) Diffusion modes

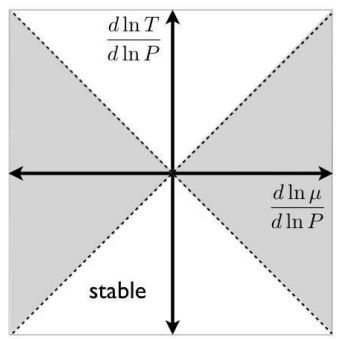

$B=B_{z} ; D \neq 0$

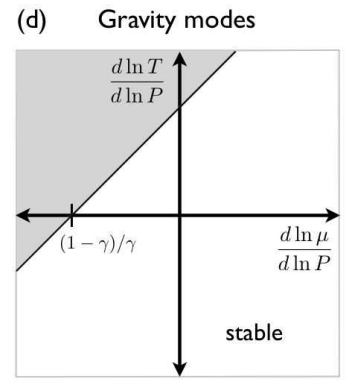

$B=B_{x}$ (e) Conduction modes

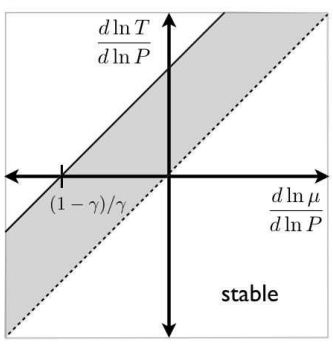

$B=B_{x}$

Figure 3. Graphic representation of the stability of modes for which conduction is slow compared to the dynamical timescale, i.e., $\omega_{\text {dyn }} \gg \tau_{\mathrm{c}}^{-1}$. The various panels show the unstable regions (gray) for each of the modes that can be excited when the background magnetic field is parallel ( $a, b$, and $c$ ) or perpendicular $(d$ and $e)$ to the background thermal and composition gradients. The solid line corresponds to $N^{2}=0$; the horizontal dotted line represents $d \ln T / d \ln P=(\gamma-1) / 2 \gamma$; and the dashed lines correspond to $d \ln T / d \ln P= \pm d \ln \mu / d \ln P$. For $B=B_{z}$, gravity modes $(a)$ can become either stable or overstable, while modes driven by conduction can become unstable (b). If ions can diffuse efficiently along magnetic field lines, a new type of mode can become unstable $(c)$. For $B=B_{x}$, both gravity modes $(d)$ and conduction modes can become unstable $(e)$; while ion-diffusion only leads to decaying modes.

of Equations (13)- 20 allows us to address the behavior of these, as well as other new modes, in the presence of a nonvanishing gradient in the mean molecular weight and account self-consistently for the diffusion of ions along magnetic field lines.

\subsection{Heat and Ion Diffusion Along Vertical Magnetic Fields}

In the case where the background magnetic field is parallel to the gravitational field, $b_{z}=1$, the dispersion relation 33 reduces to

$$
\sigma^{4}+a_{1} \sigma^{3}+a_{2} \sigma^{2}+a_{3} \sigma+a_{4}=0,
$$

with coefficients

$$
\begin{aligned}
a_{1} & =\tau_{\mathrm{c}}^{-1}+\tau_{\mathrm{d}}^{-1}+\frac{k_{\perp}^{2}}{k^{2}} \tau_{\mathrm{v}}^{-1} \approx \tau_{\mathrm{c}}^{-1} \\
a_{2} & =\frac{k_{\perp}^{2}}{k^{2}} N^{2}+\tau_{\mathrm{c}}^{-1}\left(\tau_{\mathrm{d}}^{-1}+\frac{k_{\perp}^{2}}{k^{2}} \tau_{\mathrm{v}}^{-1}\right) \approx \frac{k_{\perp}^{2}}{k^{2}} N^{2} \\
a_{3} & =\frac{k_{\perp}^{2}}{k^{2}}\left[-\tau_{\mathrm{c}}^{-1} N_{T \mu}^{2}+\tau_{\mathrm{d}}^{-1}\left(N^{2}+\tau_{\mathrm{c}}^{-1} \tau_{\mathrm{v}}^{-1}\right)\right] \\
& \approx-\tau_{\mathrm{c}}^{-1} \frac{k_{\perp}^{2}}{k^{2}} N_{T \mu}^{2} \\
a_{4} & =-\tau_{\mathrm{d}}^{-1} \tau_{\mathrm{c}}^{-1} \frac{k_{\perp}^{2}}{k^{2}} N_{T / \mu}^{2}
\end{aligned}
$$

The Routh-Hurwitz stability criteria that predict exclusively negative real parts for the roots of the quartic polynomial with real coefficients require $a_{1}>0, a_{1} a_{2}-a_{3}>0$, $a_{1} a_{2} a_{3}-a_{1}^{2} a_{4}-a_{3}^{2}>0$, and $a_{4}>0$. The first of these conditions is trivially satisfied, while the other three imply, respectively,

$$
\begin{aligned}
N_{T \mu}^{2} & <0, \\
N^{2}+N_{T \mu}^{2} & >0, \\
N_{T / \mu}^{2} & <0 .
\end{aligned}
$$

In the absence of diffusion, only the conditions 75 and (76) need to be met in order to ensure stability, while the condition (77) should also be required for finite diffusion timescales.

To leading order, two of the solutions of Equation (71) are given by $\sigma \approx \pm i a_{2}^{1 / 2}+\left(a_{3}-a_{1} a_{2}\right) / 2 a_{2}$, i.e.,

$$
\sigma \approx \pm i \frac{k_{\perp}}{k} \sqrt{N^{2}}-\frac{\tau_{\mathrm{c}}^{-1}}{2}\left(1+\frac{N_{T \mu}^{2}}{N^{2}}\right),
$$

which correspond to gravity modes. In the absence of a gradient in the mean molecular weight, these reduce to the $g$ modes discussed in Balbus \& Reynolds (2010). The third root is given by $\sigma \approx-a_{3} / a_{2}$, i.e.,

$$
\sigma \approx \tau_{\mathrm{c}}^{-1} \frac{N_{T \mu}^{2}}{N^{2}}
$$

and corresponds to a mode driven by conduction. Assuming that $N^{2}>0, g$-modes are overstable if the condition (76) is not satisfied, while conduction modes are unstable if Equation (75) is not fulfilled. The fourth solution consists of a mode driven by ion-diffusion

$$
\sigma \approx-\tau_{\mathrm{d}}^{-1} \frac{N_{T / \mu}^{2}}{N_{T \mu}^{2}},
$$

which is unstable if either criterion (75) or (77) is unfulfilled.

It is useful to understand what types of modes can be excited in the different regions of the parameter space spanned by the gradients in temperature and composition. The RouthHurwitz stability criteria take simple forms when expressed in terms of the dimensionless gradients defined in terms of the pressure. The classical requirement for stability against buoyancy, i.e., $N^{2}>0$ becomes

$$
\frac{d \ln T}{d \ln P}<\frac{d \ln \mu}{d \ln P}+\frac{\gamma-1}{\gamma},
$$

while the conditions (75) and (76) become, respectively,

$$
\begin{aligned}
& \frac{d \ln T}{d \ln P}>-\frac{d \ln \mu}{d \ln P}, \\
& \frac{d \ln T}{d \ln P}<\frac{\gamma-1}{2 \gamma} .
\end{aligned}
$$

If ions can diffuse along magnetic field lines, in addition to requiring that the gradients in temperature, pressure, and mean molecular weight satisfy the inequalities (82) and (83), the inequality 77 must also be satisfied, i.e.,

$$
\frac{d \ln T}{d \ln P}>\frac{d \ln \mu}{d \ln P} \text {. }
$$


Panel (a) in Figure 3 shows the regions of parameter space where $g$-modes in Equation (78) are stable, overstable, or unstable (gray area). Panel (b) shows that the modes in Equation (79), which are driven by conduction, can be either stable or unstable. Note that in the region of parameter space where both gravity and conduction modes are overstable/unstable they both grow with comparable rates. Panel (c) in Figure 3 shows that the modes in Equation (80), which are driven by diffusion, can be either stable or unstable (gray). Their growth rates are estimated to be an order of magnitude smaller than either $g$-modes or conduction modes. The importance of these diffusion modes resides in that they can become unstable in regions of parameter space which are stable against $g$-modes and conduction modes.

\subsection{Heat and Ion Diffusion Along Horizontal Magnetic Fields}

If the background magnetic field is perpendicular to the thermal and the composition gradients, i.e., $b_{x}=1$, the dispersion relation (33) becomes 6

$$
\sigma^{4}+b_{1} \sigma^{3}+b_{2} \sigma^{2}+b_{3} \sigma+b_{4}=0,
$$

where the coefficients

$$
\begin{aligned}
& b_{1} \approx \tau_{\mathrm{c}}^{-1}+\tau_{\mathrm{v}}^{-1} \frac{k_{\perp}^{2}}{k^{2}} \\
& b_{2} \approx N^{2} \frac{k_{x}^{2}+k_{y}^{2}}{k^{2}}+\tau_{\mathrm{c}}^{-1} \tau_{\mathrm{v}}^{-1} \frac{k_{\perp}^{2}}{k^{2}} \\
& b_{3} \approx \tau_{\mathrm{c}}^{-1} N_{T / \mu}^{2} \frac{k_{x}^{2}+k_{y}^{2}}{k^{2}}+\tau_{\mathrm{v}}^{-1} N^{2} \frac{k_{y}^{2}}{k^{2}}, \\
& b_{4} \approx \tau_{\mathrm{c}}^{-1} \tau_{\mathrm{v}}^{-1} N_{T / \mu}^{2} \frac{k_{y}^{2}}{k^{2}}
\end{aligned}
$$

are subject to the same considerations employed in deriving the approximate expressions for the coefficients $a_{i}$.

The Routh-Hurwitz stability criteria require $b_{1}>0, b_{1} b_{2}-$ $b_{3}>0, b_{1} b_{2} b_{3}-b_{1}^{2} b_{4}-b_{3}^{2}>$, and $b_{4}>0$. The first condition is trivially satisfied, while, in the limit under consideration, i.e., $\omega_{\text {dyn }} \gg \tau_{\mathrm{c}}^{-1}>\tau_{\mathrm{v}}^{-1}$, the other three conditions become, respectively,

$$
\begin{aligned}
& N^{2}-N_{T / \mu}^{2}>0, \\
& N_{T / \mu}^{2}\left(N^{2}-N_{T / \mu}^{2}\right)>0, \\
& N_{T / \mu}^{2}>0 .
\end{aligned}
$$

The inequality (90) is always satisfied, since it can be written as

$$
\frac{\gamma-1}{\gamma P \rho}\left(\frac{d P}{d z}\right)^{2}>0 .
$$

Therefore, the only independent condition required for stability is $N_{T / \mu}^{2}>0$, or $d \ln T / d z>d \ln \mu / d z$.

Two of the approximate solutions to the dispersion relation (85) are given by $\sigma \approx \pm i b_{2}^{1 / 2}+\left(b_{3}-b_{1} b_{2}\right) / 2 b_{2}$, i.e.,

$$
\sigma \approx \pm i \frac{\sqrt{k_{x}^{2}+k_{y}^{2}}}{k} \sqrt{N^{2}}-\frac{\tau_{\mathrm{c}}^{-1}}{2}\left(1-\frac{N_{T / \mu}^{2}}{N^{2}}\right) .
$$

\footnotetext{
${ }^{6}$ In the absence of diffusion, the only result that is modified in this section is that the root $\sigma=-\tau_{\mathrm{d}}^{-1}$ for $D \neq 0$ becomes $\sigma=0$ for $D=0$.
}

These correspond to gravity modes, which cannot become overstable on account of Equation 93 . The other two solutions correspond to a conduction and a viscous (decaying) mode, which are, respectively,

$$
\sigma \approx-\tau_{\mathrm{c}}^{-1} \frac{N_{T / \mu}^{2}}{N^{2}}, \quad \sigma \approx-\tau_{\mathrm{v}}^{-1} \frac{k_{y}^{2}}{k_{x}^{2}+k_{y}^{2}} .
$$

We conclude that when the magnetic field is perpendicular to the temperature and the composition gradients, the stability of $g$-modes requires only that $N^{2}>0$, whereas the stability of conduction modes requires also

$$
\frac{d \ln T}{d \ln P}<\frac{d \ln \mu}{d \ln P}
$$

This is illustrated in panel (d) in Figure 3, which shows the regions of parameter space where $g$-modes in Equation 94 are stable or unstable (gray). Panel (e) shows that the modes in Equation (95), which are driven by conduction, can be either stable or unstable. These regions are significantly different from the corresponding regions in panel (b), for which the direction of the background magnetic field is parallel to the direction of the temperature and the composition gradients. Note that, unlike the case where $b_{z}=1, g$-modes and conduction modes cannot be simultaneously unstable when $b_{x}=1$.

\section{THE PHYSICS DRIVING UNSTABLE MODES}

In previous sections we have seen that the criteria for instability result from an intricate interplay between the thermal and the composition gradients. The final expressions for the inequalities that must be satisfied for the onset of unstable modes depend implicitly on whether conduction across the associated scale is faster or slower than the dynamical timescale, the direction of the background field, and the ability of the ions to diffuse along magnetic field lines. We now analyze in detail the eigenmodes corresponding to some of the most relevant instabilities and shed light on the physical phenomena that play a role in determining the stability of the plasma. This exercise is similar in spirit to the ones presented in Quataert (2008) and Balbus (2001) to highlight the physics of the HBI and the MTI but emphasizes the effects of composition gradients and ion-diffusion along magnetic field lines. For the sake of simplicity we assume that the perturbations under consideration correspond to modes with $k_{y}=0$. In this case, the components of the Lagrangian displacement, $\partial \boldsymbol{\xi} / \partial t=\delta \boldsymbol{v}$, are related via $\xi_{x}=-\left(k_{z} / k_{x}\right) \xi_{z}$.

\subsection{Heat- and Particle-Flux Driven Buoyancy Instability}

Let us first consider a background magnetic field with $b_{z}=$ 1 and focus on perturbations with wavelengths such that the associated timescales satisfy $\tau_{\mathrm{c}}^{-1} \gg \omega_{\mathrm{dyn}} \gg \tau_{\mathrm{v}}^{-1} \simeq \tau_{\mathrm{d}}^{-1}$. For these modes, ion-diffusion along the magnetic field lines is inefficient. In order to understand the effect of a composition gradient, we retain the dominant terms in Equations 15 and (16), which leads to

$$
\delta T=\frac{d T}{d z} \xi_{z}, \quad \delta \mu=-\frac{d \mu}{d z} \xi_{z} .
$$

Because the fluctuations in density, temperature, and mean molecular weight are related via Equation (20), this implies that the relative change in density of a fluid element which is vertically displaced by $\xi_{z}$ is given by

$$
\frac{\delta \rho}{\rho}=-\left(\frac{d \ln T}{d z}+\frac{d \ln \mu}{d z}\right) \xi_{z} \quad \text { HPBI . }
$$



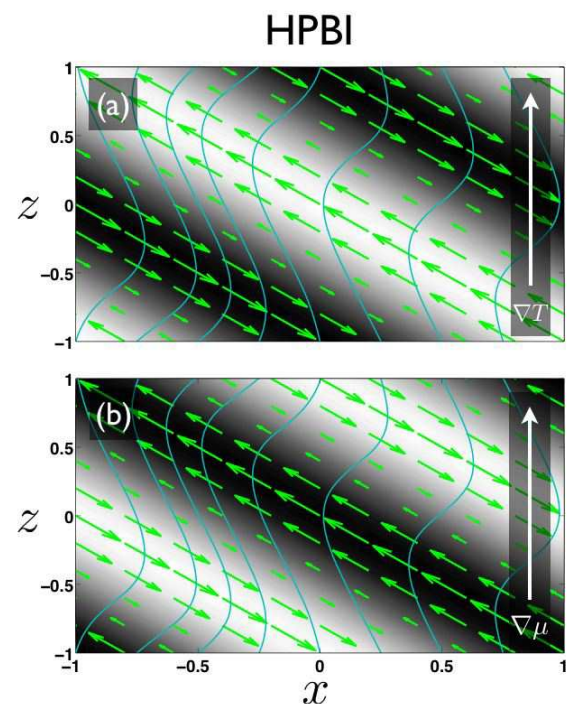

D-HPBI
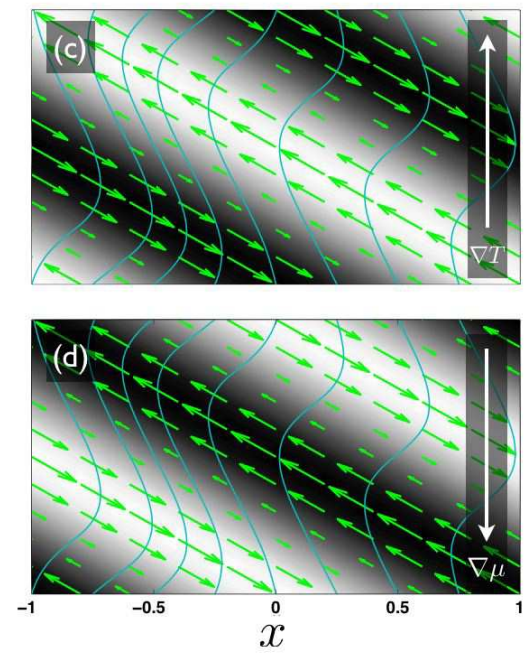

$\mathrm{MTCl}$
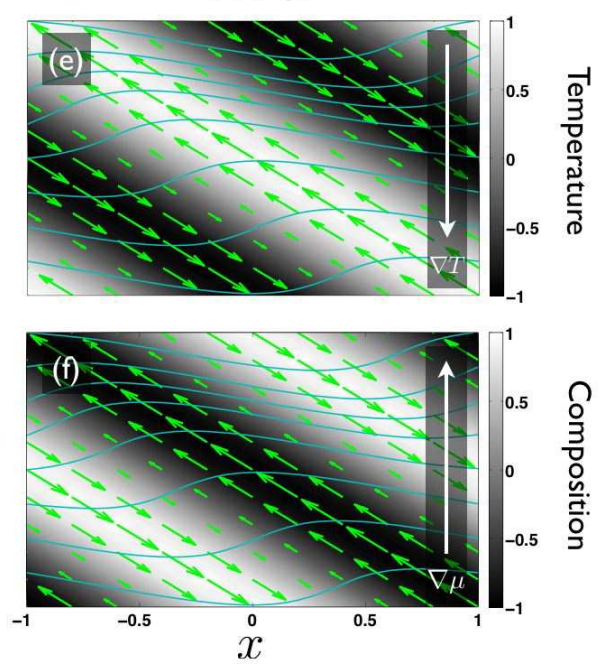

Figure 4. Schematic representation of various unstable modes in a weakly magnetized plasma with temperature and composition gradients parallel to the gravitational field $\boldsymbol{g}=-g \hat{\boldsymbol{z}}$. The three sets of panels $(\mathrm{a}, \mathrm{b}) ;(\mathrm{c}, \mathrm{d})$; and (e,f) show the modes that are unstable to the heat- and particle-flux-driven buoyancy instability (HPBI), the diffusive-HPBI, and the magneto-thermo-compositional instability (MTCI), respectively. The arrows represent the Lagrangian displacements, assumed to be of the form $\boldsymbol{\xi}=\boldsymbol{\xi}_{0} \cos \left(k_{x} x+k_{z} z\right)$, with $k_{x}=k_{z}$. The continuous lines represent the magnetic field lines, which are assumed to be parallel (HPBI, D-HPBI) or perpendicular (MTCI) to the gravitational field in the equilibrium state. The gray-scale contours show the temperature and the mean molecular weight fluctuations relative to the background gradients, which are shown with arrows indicating their directions in each of the cases considered.

It is easier to understand the physics behind this equation by analyzing first the effects of each of the two terms on the righthand side separately.

In a homogeneous medium, $d \mu / d z=0$, the term proportional to the temperature gradient is responsible for the onset of the HBI when $d T / d z>0$. This is illustrated in panel (a) in Figure 4, which shows that a fluid element that is displaced with $\xi_{z}>0\left(\xi_{z}<0\right)$ is effectively heated (cooled) by the increased (decrease) flux of heat due to the convergence (divergence) of field lines that results from the displacement of the fluid element. This causes the fluid element to expand (contract), and thus attain a density which is lower (higher) than the surrounding medium. This leads to the runaway process known as the HBI.

An isothermal environment, $d T / d z=0$, stratified in composition is unstable if $d \mu / d z>0$, as shown in panel (b) in Figure 4. Because the diffusion of ions along magnetic field lines is inefficient, the mean molecular weight of a fluid element that is displaced with $\xi_{z}>0\left(\xi_{z}<0\right)$ is lower (higher) than the surrounding medium. This results in a displaced fluid element with a density which is lower (higher) than the density of the surrounding medium, which will thus rise (sink).

If the temperature and the composition gradients are both positive (negative) then these arguments act in consonance and lead to the conclusion that the plasma is unstable (stable). On the other hand, if the temperature and the composition gradients have different signs it follows that if $d T / d z>0$ $(d \mu / d z>0)$ is steep enough then the expansion induced in an upwardly displaced fluid element can offset the stabilizing effects of $d \ln \mu / d z<0(d \ln T / d z<0)$ and the plasma will be unstable, giving rise to an HPBI.

The arguments outlined here can also be derived from the equation of motions for the Lagrangian displacement $\xi_{z}$. The buoyancy force per unit volume on a vertically displaced fluid element produces an acceleration given by

$$
\frac{d^{2} \xi_{z}}{d t^{2}}=-g \frac{\delta \rho}{\rho}
$$

and thus, according to Equation 98 ,

$$
\frac{d^{2} \xi_{z}}{d t^{2}}=N_{T \mu}^{2} \xi_{z}
$$

This leads to an instability if $N_{T \mu}^{2}>0$, in agreement with the results of Section 4 .

\subsection{Diffusive Heat- and Particle-Flux Driven Buoyancy Instability}

The effects of ion-diffusion are not negligible for the modes for which the associated timescales satisfy $\tau_{\mathrm{c}}^{-1}>\tau_{\mathrm{v}}^{-1} \simeq$ $\tau_{\mathrm{d}}^{-1} \gg \omega_{\mathrm{dyn}}$. To leading order, the changes in the temperature and the mean molecular weight in a fluid element which is vertically displaced by $\xi_{z}$ in a background magnetic field with $b_{z}=1$ are given by

$$
\delta T=\frac{d T}{d z} \xi_{z}, \quad \delta \mu=\frac{d \mu}{d z} \xi_{z} .
$$

Note that because the dominant terms in Equation (16) are both proportional to $D$, the fractional change in the mean molecular weight is independent of the value of the diffusion coefficient. The fractional change in density is thus

$$
\frac{\delta \rho}{\rho}=-\left(\frac{d \ln T}{d z}-\frac{d \ln \mu}{d z}\right) \xi_{z} \quad \text { D-HPBI . }
$$

The role played by the background temperature gradient is identical to the one discussed in the absence of ion-diffusion, and this situation is shown for the sake of clarity in panel (c) in Figure 4 However, the contribution from the mean molecular weight gradient is now the opposite. This difference in sign is due to the manifest role played by ion-diffusion as an effective agent to tap into the free energy available in the background particle flux needed to maintain the composition gradient.

In order to understand the role played by ion-diffusion let us focus on a background with constant temperature, $d T / d z=$ 
0 . The term proportional to the composition gradient is responsible for the onset of the D-HPBI when $d \mu / d z<0$. This is illustrated in panel (d) in Figure 4, which shows that the mean molecular weight of a fluid element that is displaced with $\xi_{z}>0\left(\xi_{z}<0\right)$ will decrease (increase) because of the decrease (increase) in the flux of particles out of (into) it due to the diverge (convergence) of field lines that results from the displacement of the fluid parcel. This causes the density of the fluid element to decrease (increase) with respect to the surrounding medium, leading to a runaway process. A background temperature gradient with $d T / d z>0$ will reinforce this process leading to enhanced buoyancy. In general, when both the temperature and the composition gradients are non-zero, the condition for D-HPBI is $d \ln T / d z<d \ln \mu / d z$. This is reflected in the equation of motion for the Lagrangian displacement

$$
\frac{d^{2} \xi_{z}}{d t^{2}}=N_{T / \mu}^{2} \xi_{z}
$$

which has exponentially growing solutions when $N_{T / \mu}^{2}>0$, as we have seen before.

\subsection{Magneto-Thermo-Compositional Instability}

Let us now consider a background horizontal magnetic field with $b_{x}=1$ and modes for which conduction is faster than any other timescale. When a fluid element is displaced by $\xi_{z}$ from its equilibrium position, the fluctuations in temperature and composition are given by

$$
\delta T=-\frac{d T}{d z} \xi_{z}, \quad \delta \mu=-\frac{d \mu}{d z} \xi_{z}
$$

It is important to note that this relationship between the fractional change in the mean molecular weight and the Lagrangian displacement holds regardless of whether the dynamical timescale is fast or slow with respect to the viscous and diffusion timescales. The only difference is that in the former case the leading order terms in Equation (16) that lead to $\delta \mu=-(d \mu / d z) \xi_{z}$ are independent of the coefficient $D$, while in the opposite limit, the dominant terms are those proportional to $D$. In either case, the relative change in density becomes

$$
\frac{\delta \rho}{\rho}=\left(\frac{d \ln T}{d z}-\frac{d \ln \mu}{d z}\right) \xi_{z} \quad \text { MTCI } .
$$

In this case, the term proportional to the temperature gradient is responsible for the onset of the MTI when $d T / d z<0$ in a homogeneous medium, i.e., $d \mu / d z=0$. In panel (e) in Figure 4 we consider the situation where $\tau_{d}^{-1} \gg \omega_{\text {dyn }}$. Under this condition, a fluid element that is displaced with $\xi_{z}>0$ $\left(\xi_{z}<0\right)$ is effectively heated (cooled) by conduction along the magnetic field lines which have been distorted by the displacement of the fluid element. This causes the fluid element to expand (contract), and thus attain a density which is lower (higher) than the surrounding medium. This leads to the runaway process known as the MTI. The term proportional to the composition gradient is responsible for the onset of the MTCI when $d \mu / d z>0$ in an isothermal environment. This is illustrated in panel (f) in Figure 4 which shows that because of the effective diffusion of ions along distorted magnetic field lines, fluid elements that have been displaced upward (downward) $\xi_{z}>0\left(\xi_{z}<0\right)$ maintain the mean molecular weight corresponding to the equilibrium value at their original position. This implies that the fluid element is immersed in a medium that is relatively denser (lighter) and it will thus rise (sink).
When both gradients are non-zero their relative magnitudes determine whether the plasma is buoyantly unstable according to Equation (105). This can also be seen in the equation of motion

$$
\frac{d^{2} \xi_{z}}{d t^{2}}=-N_{T / \mu}^{2} \xi_{z}
$$

which has exponentially growing solutions when $N_{T / \mu}^{2}<0$.

\subsection{Overstable Modes}

The physics driving overstable modes, which are present when $b_{z}=1$, is more subtle, but some insight can be gained by analyzing directly the equations of motion for the corresponding Lagrangian displacements. Using Equations 57 and (78) together with the equations for the perturbations 13)-116 we find, to first order,

$\frac{d^{2} \xi_{z}}{d t^{2}}=N_{T \mu}^{2} \xi_{z}-\frac{N^{2}+N_{T \mu}^{2}}{\tau_{\mathrm{c}}^{-1}} \frac{d \xi_{z}}{d t}, \quad\left(\tau_{\mathrm{c}}^{-1} \gg \omega_{\mathrm{dyn}}\right) ;$
$\frac{d^{2} \xi_{z}}{d t^{2}}=-N^{2} \xi_{z}-\tau_{\mathrm{c}}^{-1} \frac{N^{2}+N_{T \mu}^{2}}{N^{2}\left(k_{\perp}^{2} / k^{2}\right)} \frac{d \xi_{z}}{d t},\left(\omega_{\text {dyn }} \gg \tau_{\mathrm{c}}^{-1}\right)$.

The physics behind the first terms on the right-hand side of each of these equations is readily recognized. The term proportional to $N_{T \mu}^{2}$ is responsible for the HPBI if $N_{T \mu}^{2}>0$, while the term proportional to $N^{2}$ is responsible for BruntViäsälä oscillations, i.e., stable $g$-modes, if $N^{2}>0$. In a medium where $N_{T \mu}^{2}<0$ and $N^{2}>0$ but $N^{2}+N_{T \mu}^{2}<0$, the anisotropic heat flow along magnetic field lines tends to monotonically increase the restoring force acting on the oscillating fluid parcel and leads to overstability.

\section{ASTROPHYSICAL IMPLICATIONS}

Throughout this paper, we have studied the various instabilities that can be present in the parameter space spanned by $(d \ln \mu / d \ln P, d \ln T / d \ln P)$ without imposing restrictions on the relative values of the gradients involved. We can now frame our results in the context provided by observations and theoretical models addressing the temperature and composition structure of galaxy clusters.

\subsection{Temperature and Composition Profiles in the ICM from Models and Observations}

Modern X-ray observatories, such as Chandra and XMMNewton, have made it possible to obtain the temperature profiles of a large number of galaxy clusters, see, e.g., Vikhlinin et al. (2006); Leccardi \& Molendi (2008). This has enabled to show that, quite generically, the gas temperature increases with radius, reaching values of $5-10 \mathrm{keV}$ at 100 $\mathrm{kpc}$, and decreases toward the outer cluster regions. In several cases the temperature changes by a factor of two or three on distances comparable to the cluster radius.

In spite of the small mass ratio between hydrogen and helium, because helium is relatively abundant in the primordial gas from which clusters form, its sedimentation over the life-time of the cluster has the potential to produce important gradients in the mean molecular weight profile. Obtaining observational evidence to quantify the helium abundances that would result form this sedimentation is very hard because helium is completely ionized at the characteristic temperatures of typical galaxy clusters. However, this information is key in order to derive physical cluster 


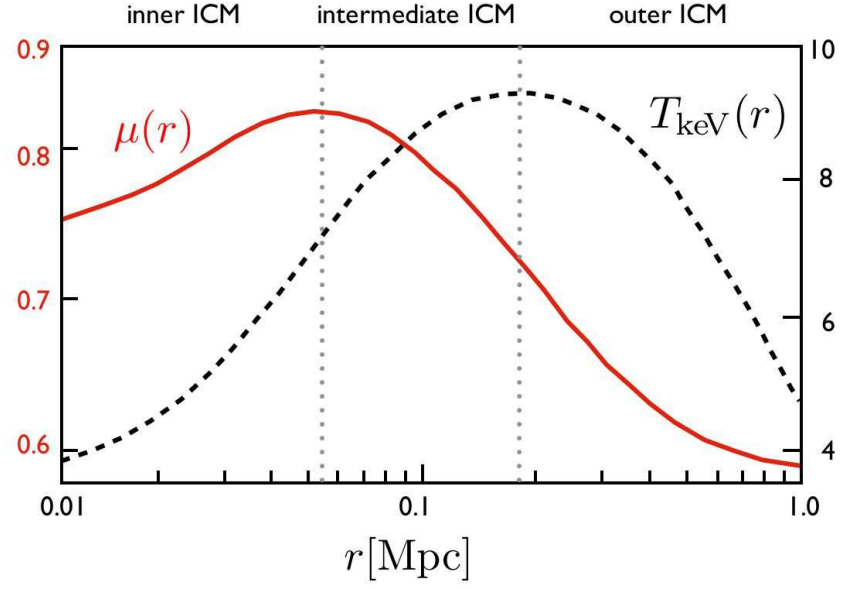

Figure 5. Schematic representation of the mean molecular weight (red solid line) and temperature (black dashed line) profiles of a representative galaxy cluster as suggested by observations (Vikhlinin et al. 2006) and theoretical models (Bulbul et al. 2011). The regions denoted by "inner", "intermediate", and "outer" ICM (delimited by dotted gray lines) correspond to three different quarters in the $(d \ln \mu / d \ln P, d \ln T / d \ln P$ ) plane (Figures 23 and panel (a) in Figure 6. The mean molecular weight for a homogeneous cluster with primordial abundance is $\mu \simeq 0.6$

properties, such as gas mass, total mass and gas mass fraction (see, e.g., Oin \& Wu 2000), and even cluster distances (Markevitch 2007) from X-ray observations. This has highlighted the need to understand the efficiency of this process on theoretical and observational grounds (Fabian \& Pringle 1977; Abramopoulos et al. 1981; Gilfanov \& Syunvaev 1984; Qin \& Wu 2000; Chuzhoy \& Nusser 2003; Chuzhoy \& Loeb 2004; Tamura et al. 2004; Ettori \& Fabian 2006; Peng \& Nagai 2009). Some of these estimates predict an overabundance by up to a factor of a few with respect to the primordial value $\mu \simeq 0.6$.

Since the realization that the stability properties of a weakly magnetized medium depends critically on its thermal structure, a large number of works have been devoted to study the HBI and the MTI. In spite of the fact that both instabilities grow on a dynamical timescale, the onset of the most relevant modes depends explicitly on the local values of the thermal gradients. Throughout this study, we have seen that if the composition of the plasma is not homogeneous, it is the combination of both the thermal and the mean molecular weight gradients that decides whether the plasma is stable or not. In order to understand how important the contributions from each of these gradients can be, we consider the schematic representation of the temperature and the mean molecular weight profiles of the representative galaxy cluster shown in Figure 5 The temperature profile shown resembles the results obtained by observations (Vikhlinin et al. 2006), whereas the mean molecular weight profile is akin to the helium sedimentation models discusses in Bulbul et al. (2011), which are based on analytical models for the physical properties of the ICM introduced in Bulbul et al. (2010). Although very crude, these representative profiles allow us to provide an estimate for the values of the logarithmic gradients for the temperature, $(\nabla T) / T$, and the mean molecular weight, $(\nabla \mu) / \mu$, which play a role in determining the stability of the dilute ICM. If the peak in the temperature profile occurs at a larger radius than the peak in the mean molecular weight profile, as shown in Figure 5 then there are three distinct regions defined by the signs of the temperature and the composition gradients. Each of these regions of the ICM will have asso- ciated different characteristic values, which can be estimated according to

$$
\frac{\nabla T}{T} \simeq \frac{1}{L} \frac{\Delta T}{\bar{T}}, \quad \frac{\nabla \mu}{\mu} \simeq \frac{1}{L} \frac{\Delta \mu}{\bar{\mu}} .
$$

Here, $\Delta T \equiv T_{\text {out }}-T_{\text {in }}$ stands for the difference between two values across the characteristic scale $L \equiv r_{\text {out }}-r_{\text {in }}$, and $\bar{T} \equiv\left(T_{\text {out }}+T_{\text {in }}\right) / 2$ is the associated mean value, with similar definitions for the mean molecular weight. Using the information available in Figure 5, we estimate these characteristic values for the different ICM regions in Section 7.3 .

\subsection{Applicability of Approximations in the ICM}

Before addressing the stability of the different regions of the ICM, we comment on two of the approximations that we have made on the geometry and strength of the magnetic field, which have allowed us to gain physical insight while keeping the problem tractable.

We have studied in detail two special cases for the orientation of the background magnetic field, viz., either parallel, $\boldsymbol{B}=B \hat{\boldsymbol{z}}$, or perpendicular, $\boldsymbol{B}=B \hat{\boldsymbol{x}}$, to the gravitational field $\boldsymbol{g}$. These two configurations, which have received a lot of attention in the related literature, have the advantage of not only simplifying the mathematics involved but also exposing in a clean way the physics driving the HBI, the MTI, as well as the generalizations that result from including composition gradients. While beyond the scope of this paper, accounting for more general geometries is clearly necessary in order to describe more realistic situations.

An important simplification in our study is the assumption that the magnetic field is so weak that the Alfvén frequency is much smaller than any other inverse timescale involved. It is worth mentioning that this is the regime explored by a number of numerical studies addressing both fundamental aspects of the MTI and the HBI (Parrish \& Stone 2005, 2007; Parrish \& Ouataert 2008; Latter \& Kunz 2012), as well as the implications that these instabilities have for the long-term evolution of the ICM (Bogdanović et al. 2009; Ruszkowski \& Oh 2010; Parrish et al. 2008, 2009, 2010, 2012; McCourt et al. 2011, 2012; Kunz et al. 2012). For the modes for which magnetic tension cannot be neglected, the Alfvén timescale can become comparable or even faster than the dynamical and the conduction timescales and neglecting $\omega_{\mathrm{A}}$ in the dispersion relation (33) is not a good approximation.

For the modes for which magnetic tension is important, the explicit dependence on $\omega_{\mathrm{A}}$ might introduce new stability criteria which have not been captured by our analysis. Furthermore, magnetic tension could affect the growth rates of the instabilities. Kunz (2011) provides a summary of the stabilizing effects provided by magnetic tension on HBI- and MTIunstable modes in a homogeneous medium. The main physical effect introduced by a non-zero Alfvén frequency is to provide a cut-off for the growth of unstable modes at parallel wavenumbers such that $k_{\|} v_{\mathrm{A}}$ is comparable to the growth rate of the most unstable modes. For the magnetic field geometries that we analyzed, this must also be the case even in the presence of a composition stratification. The reason for this is that, when either $b_{x}=1$ or $b_{z}=1$, all the contributions introduced by the mean molecular weight gradient appear in the form $d \ln T / d z \pm d \ln \mu / d z$. Thus, while the growth rates and range of unstable modes are affected because of the changes in the background composition, the effects of non-negligible magnetic tension on these modes, i.e., the existence of a cut-off parallel wavenumber, must be similar to what has been found in the case of a homogeneous medium. Since the plasma $\beta$ 
Table 1

Representative Parameter Values for Different ICM Regions

\begin{tabular}{lccccccc}
\hline \hline ICM Region & $\nabla T$ & $\nabla \mu$ & $\beta$ & $K_{n}^{-1}$ & $\begin{array}{c}\text { Local Modes in Fluid Model } \\
K_{n}^{1 / 2}<\tilde{k}_{\|}<K_{n}^{-1 / 2}\end{array}$ & $\begin{array}{c}\text { Fast Conduction and } \omega_{\mathrm{A}}<\omega_{\text {dyn }} \\
1 / 3<\tilde{k}_{\|}<\left(\beta K_{n}\right)^{1 / 2}\end{array}$ & $\begin{array}{c}\text { Slow Conduction and } \omega_{\mathrm{A}}<\tau_{\mathrm{c}}^{-1} \\
0.1\left(\beta K_{n}\right)^{-1 / 2}<\tilde{k}_{\|}<1 / 3\end{array}$ \\
\hline Outer-ICM & $<0$ & $<0$ & $10^{4}$ & $10^{1}$ & $0.3<\tilde{k}_{\|}<3$ & $1 / 3<\tilde{k}_{\|}<30$ & $0.003<\tilde{k}_{\|}<1 / 3$ \\
Interm.-ICM & $>0$ & $<0$ & $10^{3}$ & $10^{2}$ & $0.1<\tilde{k}_{\|}<10$ & $1 / 3<\tilde{k}_{\|}<3$ & $0.03<\tilde{k}_{\|}<1 / 3$ \\
Inner-ICM & $>0$ & $>0$ & $10^{2}$ & $10^{3}$ & $0.03<\tilde{k}_{\|}<30$ & $1 / 3<\tilde{k}_{\|}<0.3$ & $0.3<\tilde{k}_{\|}<1 / 3$ \\
\hline
\end{tabular}

Note. - The various ICM regions are defined in Figure 5 For convenience, in this table we have defined the dimensionless parallel wavenumber, $\tilde{k}_{\|}=k_{\|}\left(\lambda_{\mathrm{mfp}} H\right)^{1 / 2}$.

in galaxy clusters varies with radius, whether neglecting magnetic tension is a sensitive approximation for a local stability analysis or not, depends on the conditions present in the region of the ICM under consideration. We address this issue in detail below.

\subsection{Stability of ICM regions}

The analysis of Figures 2 and 3 allows us to understand the implications that a mean molecular weight gradient can have for the various regions of a representative galaxy cluster as depicted in Figure 5. These regions correspond to different quadrants in the $(d \ln \mu / d \ln P, d \ln T / d \ln P)$ plane as shown in panel (a) of Figure 6, which we have denoted as inner, intermediate, and outer ICM. In what follows we will assume, as suggested by observations of galaxy clusters, that the ICM is buoyantly stable according to the classical stability criterion $N^{2}>0$ (Piffaretti et al. 2005; Cavagnolo et al. 2009).

\subsubsection{Outer ICM}

In this region the temperature and the mean molecular weight gradients are both negative. Because the criteria for stability are different whether conduction is fast or slow compared to the dynamical timescale, we consider these two cases separately.

Fast conduction. If the magnetic field lines are parallel to the gravitational field, i.e., $b_{z}=1$, gravity modes can become overstable if $d \ln T / d \ln P>(\gamma-1) /(2 \gamma)$, panel (a) in Figure 2. A gradient in the mean molecular weight alone is unable to stabilize these modes and can drive unstable modes driven by diffusion if $|\nabla \mu| / \mu>|\nabla T| / T$, panels (b) and (c) in Figure 2. For magnetic field configurations that are perpendicular to the gravitational field, i.e., $b_{x}=1$, this region is unstable to the MTCI provided that $|\nabla \mu| / \mu<|\nabla T| / T$, panel (d) in Figure 2. This means that the outskirts of galaxy clusters that would be considered prone to the MTI (if they were homogeneous) would remain stable if the gradient in mean molecular weight is steep enough. This is not the case for the particular profiles shown in Figure 5 but this does not imply that this is not the case in general.

Slow conduction. In the absence of ion-diffusion, gravity modes can become overstable if $\mathrm{bz}=1$. These modes cannot be stabilized by means of a gradient in the mean molecular weight alone, panel (a) in Figure 3 . Furthermore, when iondiffusion is efficient, it can drive unstable modes if $|\nabla \mu| / \mu>$ $|\nabla T| / T$, panel (c) in Figure 3. For $b_{x}=1$ there can be unstable modes driven by conduction if $|\nabla \mu| / \mu<|\nabla T| / T$, panel (d) in Figure 3 .

We can provide a crude estimate of the impact that a composition gradient would have on the growth rates of the various instabilities discussed by estimating the temperature and the composition gradients shown in Figure 5. For the inner
ICM region, the characteristic scale is $L \simeq 0.8 \mathrm{Mpc}$, while $\Delta T \simeq 4 \mathrm{keV}, \bar{T} \simeq 7 \mathrm{keV}, \Delta \mu \simeq 0.1$, and $\bar{\mu} \simeq 0.65$. Thus according to Equations 109, the characteristic logarithmic gradients in this inner region are given by

$$
\left.\frac{\nabla T}{T}\right|_{\text {outer }} \simeq-0.7 \mathrm{Mpc}^{-1},\left.\frac{\nabla \mu}{\mu}\right|_{\text {outer }} \simeq-0.2 \mathrm{Mpc}^{-1} \text {. }
$$

These order-of-magnitude estimates, based on the representative values drawn from Figure 5, show that the instabilities with growth rates $\sigma^{2} \propto-\ln (T / \mu)$, such as the generalization of the MTI, Equations (59) and (68), will be $15 \%$ slower with respect to the homogeneous case.

Regarding the validity of our assumptions of weak magnetic fields, panel (b) of Figure 6 shows that magnetic tension is unimportant for the range of modes of interest, i.e., $K_{\mathrm{n}}^{1 / 2}<k_{\|}\left(\lambda_{\mathrm{mfp}} H\right)^{1 / 2}<K_{\mathrm{n}}^{-1 / 2}$. Thus, our approximation of setting $\omega_{\mathrm{A}} \simeq 0$ is fully justified in this region.

\subsubsection{Intermediate ICM}

In this region the temperature and the mean molecular weight gradients have different signs, $\square$ with $\nabla \mu<0$ and $\nabla T>0$ according to the profiles shown in Figure 5.

When $b_{z}=1$, this region is unstable due to the HPBI, which grows on the dynamical timescale if $|\nabla \mu| / \mu<$ $|\nabla T| / T$. This instability can be prevented if the mean molecular weight is steep enough, panel (a) in Figure 2 When ion-diffusion is efficient this region is unstable due to the D-HPBI, panel (c) in Figure 2. This magnetic field configuration is also prone to unstable modes for which conduction is slow. In this case there are unstable modes driven by conduction if $D=0$ and $|\nabla \mu| / \mu<|\nabla T| / T$, while there are unstable modes driven by ion-diffusion if $D \neq 0$ and $|\nabla \mu| / \mu>|\nabla T| / T$, panels (b) and (c) in Figure 2] respectively. If $b_{x}=1$, this region is stable whether conduction is fast or slow compared to the dynamical timescale, panels (d) in Figure 2 and (e) in Figure 3.

For this intermediate ICM region, the inspection of Figure 5 provides $L \simeq 0.15 \mathrm{Mpc}, \Delta T \simeq 2 \mathrm{keV}, \bar{T} \simeq 8 \mathrm{keV}, \Delta \mu \simeq 0.1$, and $\bar{\mu} \simeq 0.75$. Thus the characteristic logarithmic gradients are given by

$$
\left.\frac{\nabla T}{T}\right|_{\text {interm. }} \simeq 1.6 \mathrm{Mpc}^{-1},\left.\frac{\nabla \mu}{\mu}\right|_{\text {interm. }} \simeq-1 \mathrm{Mpc}^{-1} \text {. }
$$

Therefore, the instabilities with growth rates for which $\sigma^{2} \propto$ $\ln (T \mu)$, such as the generalization of the HBI in the absence

\footnotetext{
${ }^{7}$ Note that if the peak in $\mu$ were to occur beyond the radius at which the temperature is maximum, then this region would be characterized by $d T / d z<0$ and $d \mu / d z>0$, which corresponds to the opposite quadrant in panel (a) of Figure 6
} 
(a)

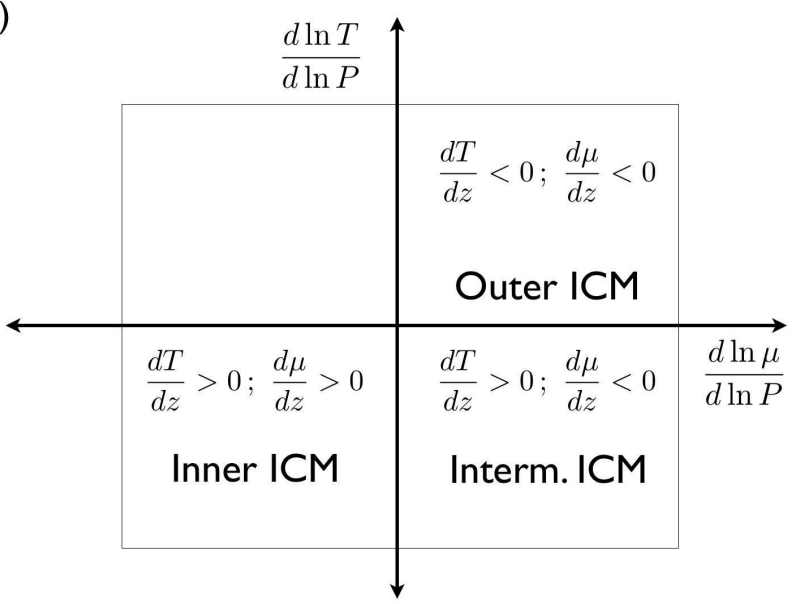

(d) Inner ICM

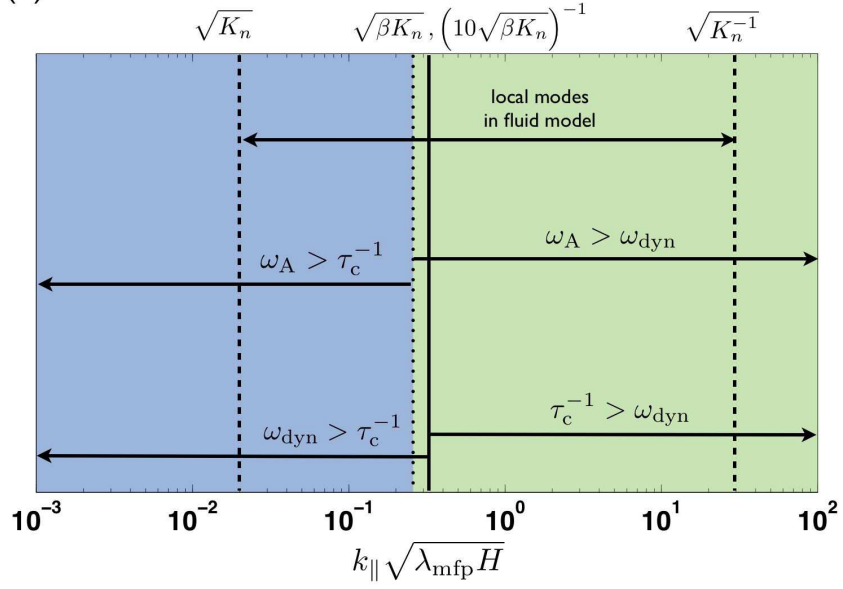

(b) Outer ICM

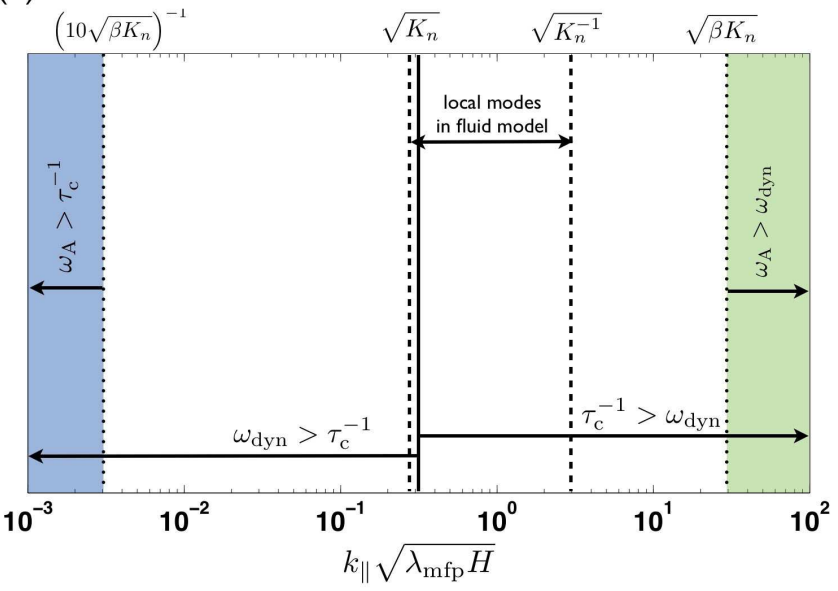

(c) Intermediate ICM

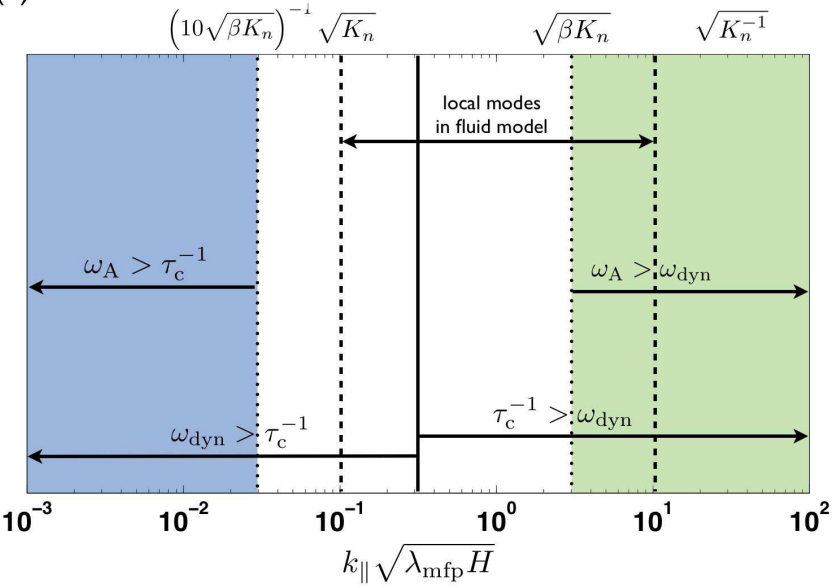

Figure 6. Panel (a) shows a schematic representation of the correspondence between the regions of a representative galaxy cluster with the radial temperature and the mean molecular weight profiles as shown in Figures 5 and the plane spanned by $(d \ln T / d \ln P, d \ln \mu / d \ln P)$. The various vertical lines in panels (b)-(d) mark some relevant values of the dimensionless parallel wavenumber, $k_{\|}\left(\lambda_{\mathrm{mfp}} H\right)^{1 / 2}$. The region between the dashed lines corresponds to the range of local modes for which the fluid approximation is valid, i.e., $K_{\mathrm{n}}^{1 / 2}<k_{\|}\left(\lambda_{\mathrm{mfp}} H\right)^{1 / 2}<K_{\mathrm{n}}^{-1 / 2}$. The solid line, at $k_{\|}\left(\lambda_{\mathrm{mfp}} H\right)^{1 / 2}=1 / 3$, distinguishes between modes for which conduction timescale is smaller (to the right) or larger (to the left) than the dynamical timescale. The dotted lines represent the value of the $k_{\|}\left(\lambda_{\mathrm{mfp}} H\right)^{1 / 2}$ beyond which magnetic tension cannot be neglected. This corresponds to $k_{\|}\left(\lambda_{\mathrm{mfp}} H\right)^{1 / 2}>\left(\beta K_{n}\right)^{1 / 2}$ (green) for fast conduction modes, and $k_{\|}\left(\lambda_{\mathrm{mfp}} H\right)^{1 / 2}<\left(\beta K_{n}\right)^{-1 / 2} / 10$ (blue) for slow conduction modes. All the numerical values associated with the various length scales and timescales have been drawn from Table 1 which provides estimates for the $\beta$ plasma and the Knudsen number representative of the different regions of the ICM.

of ion-diffusion, Equation (54), will grow of the order of $40 \%$ slower with respect to the homogeneous case. On the other hand, the instabilities with growth rates $\sigma \propto \ln (T / \mu)$, such as the generalization of the HBI with ion-diffusion, Equation (64), will be $30 \%$ faster.

As shown in panel (c) in Figure 6 magnetic tension is important for some of the modes for which conduction is fast but not for the modes for which conduction is slow.

\subsubsection{Inner ICM}

In this region the temperature and the mean molecular weight gradients are both positive. We consider again the limits in which conduction is fast or slow separately.

Fast conduction. For $b_{z}=1$, this region is unstable to the HPBI regardless of whether the mean molecular weight gradient is smaller or larger than the temperature gradient, panel (a) in Figure 2. Furthermore, if ion-diffusion is efficient it can also drive unstable modes, panels (b) and (c) in Figure 2, In a homogeneous medium with $b_{x}=1$, this inner region is sta- ble against the MTI. However, there can be unstable MTCImodes if $|\nabla \mu| / \mu>|\nabla T| / T$, panel (d) in Figure 2 In the homogeneous case when $\nabla T>0$, the HBI tends to re-orient the magnetic field in the radial direction, which results in a field configuration which is stable against the MTI, i.e., $b_{x} \simeq 1$. When ion-diffusion is not efficient, this core insulation could be alleviated by the MTCI if the mean molecular weight gradient is steep enough, i.e., $(\nabla \mu) / \mu>(\nabla T) / T$.

Slow conduction. This region can be subject to instabilities driven by both heat conduction and ion-diffusion. When $b_{z}=1$ and ion-diffusion is inefficient, there are unstable modes driven by heat conduction regardless of the relative magnitude of the temperature and the mean molecular weight profiles, panel (b) in Figure 3, whereas there are unstable modes driven by diffusion if $|\nabla \mu| / \mu>|\nabla T| / T$, panel (c) in Figure 3. In the case with $b_{x}=1$, there can be unstable modes driven by heat conduction if $|\nabla \mu| / \mu>|\nabla T| / T$, panel (e) in Figure 3.

According to Figure [5] the inner ICM region is charac- 
terized by $L \simeq 0.05 \mathrm{Mpc}, \Delta T \simeq 3 \mathrm{keV}, \bar{T} \simeq 5.5 \mathrm{keV}$, $\Delta \mu \simeq 0.1$, and $\bar{\mu} \simeq 0.8$, and thus

$$
\left.\frac{\nabla T}{T}\right|_{\text {inner }} \simeq 10 \mathrm{Mpc}^{-1},\left.\frac{\nabla \mu}{\mu}\right|_{\text {inner }} \simeq 2.5 \mathrm{Mpc}^{-1} .
$$

Therefore, the instabilities with growth rates for which $\sigma^{2} \propto$ $\ln (T \mu)$ will grow of the order of $10 \%$ faster with respect to the homogeneous case, while the instabilities with growth rates $\sigma^{2} \propto \ln (T / \mu)$ will be $15 \%$ slower.

The analysis of Figure 6 shows that the range of modes for which it is sensible to carry out a local mode analysis within the fluid model embodied in Equations (1)-(5) increases as the inverse Knudsen number increases toward smaller radii. However, due to the increase in the strength of the background magnetic field, the range of modes for which it is sensible to neglect magnetic tension, decreases. Because the relatively low values of $\beta$ in the inner core region, magnetic tension is important for all the modes of interest. Therefore, our conclusions for this region should be considered with caution.

\subsubsection{Summary and Outlook}

During the last few years, there has been substantial numerical work for understanding the long-term evolution of the MTI and the HBI and their implications for the gas dynamics in the ICM permeating galaxy clusters (Bogdanović et al. 2009; Parrish et al. 2008, 2009, 2010, 2012; McCourt et al. 2011, 2012; Kunz et al. 2012). All of this work has been done under the assumption that the ICM is homogeneous and thus the temperature gradient provides the only source of energy to feed instabilities. Even though it is hard to quantify concentration gradients from observations, some heavy element sedimentation is expected (Narayan \& Medvedev 2001; Chuzhoy \& Nusser 2003; Chuzhoy \& Loeb 2004; Ettori \& Fabian 2006). Indeed, current theoretical models suggest that helium sedimentation can significantly alter the composition profile throughout the cluster and give rise to mean molecular weight gradients which are comparable in magnitude to the temperature gradients, with $|\nabla T| / T \simeq|\nabla \mu| / \mu$ (see, e.g., Bulbul et al. 2011, and Figure 5].

This work discusses for the first time the effects that composition gradients can have for the stability of a weakly collisional magnetized medium which is stratified in both temperature and composition. We have found that, depending on the wavelength of the modes under consideration, the plasma can be subject to a wide variety of unstable modes. These include:

- the generalization of the MTI (Balbus 2001):

$$
\sigma^{2} \approx-g \frac{d \ln (T / \mu)}{d z} \frac{k_{x}^{2}+k_{y}^{2}}{k^{2}}
$$

and the generalization of the HBI (Quataert 2008) in

- the slow ion-diffusion limit:

$$
\sigma^{2} \approx g \frac{d \ln (T \mu)}{d z} \frac{k_{\perp}^{2}}{k^{2}}
$$

- the fast ion-diffusion limit:

$$
\sigma \approx \frac{g}{\tau_{\mathrm{v}}^{-1}} \frac{d \ln (T / \mu)}{d z}
$$

We have also found the generalization of the overstable gravity modes discussed in Balbus \& Reynolds (2010), see Equations (57) and (78), as well as other new modes which are driven by conduction and diffusion.

This study constitutes a first step toward the long-sought goal of understanding in a self-consistent way the effects of magnetic turbulence on the diffusion of heavy elements and its consequences for the observational signatures and longterm evolution of galaxy clusters. This will only be possible through numerical studies involving realistic models for the microphysics of weakly collisional, multi-component plasmas. Addressing this problem will require to sort out several details, including how to properly handle plasma microinstabilities, many of which are still the subject of active research in homogeneous settings.

We thank Matthew Kunz, Henrik Latter, Aldo Serenelli, and Shantanu Mukherjee for useful discussions. We are grateful to the anonymous referee for a detailed and thoughtful report that helped us improve the final version of this manuscript significantly. M.E.P is grateful to the Knud Højgaard Foundation and the Villum Foundation for their generous support. S.C. acknowledges support from the Danish Research Council through FNU Grant No. 505100-50 - 30,168.

\section{APPENDIX}

\section{A. ION DIFFUSION IN A BINARY MIXTURE}

The ratio between the timescales associated with viscous and diffusion processes is $\tau_{\mathrm{v}}^{-1} / \tau_{\mathrm{d}}^{-1}=3 \nu_{\|} / D$. Here, the coefficient $\nu_{\|}=v_{\text {th }}^{2} /\left(2 \nu_{i i}^{\text {eff }}\right)$ denotes the kinematic Braginskii viscosity associated with a binary mixture of ions, where $\nu_{i i}^{\text {eff }}$ is an effective ion-ion collision rate which can be estimated as follows. The Braginskii viscosity for a single species of ions is $\eta_{0}=\rho v_{\text {th }}^{2} /\left(2 \nu_{i i}\right)$, where $\nu_{i i}$ is the ion-ion collision frequency

$$
\nu_{i i}=\frac{4 \sqrt{\pi}}{3} \frac{n_{i} q_{i}^{4}}{m_{i}^{1 / 2}\left(k_{B} T\right)^{3 / 2}} \ln \Lambda_{i i} .
$$

Here $n_{i}, m_{i}$ and $q_{i}$ are the number density, the mass, and the charge of the ion respectively; and $\ln \Lambda$ refers to the corresponding Coulomb logarithm. In a binary mixture, the effective viscosity coefficient (ignoring the contribution from electrons) is given by

$$
\eta_{0} \simeq \frac{n_{i_{1}} k_{B} T}{\nu_{i_{1} i_{1}}+\nu_{i_{1} i_{2}}}+\frac{n_{i_{2}} k_{B} T}{\nu_{i_{2} i_{1}}+\nu_{i_{2} i_{2}}}
$$

where the collision frequency between ions of species $i$ and $j$ is

$$
\nu_{i j}=\frac{4 \sqrt{2 \pi}}{3}\left[\frac{\sqrt{m_{i j}} q_{i}^{2} q_{j}^{2} n_{j}}{m_{i}\left(k_{B} T\right)^{3 / 2}}\right] \ln \Lambda_{i j},
$$

and $m_{i j} \equiv m_{i} m_{j} /\left(m_{i}+m_{j}\right)$ is the reduced ion mass. We can thus define the effective ion-ion collision frequency as

$$
\nu_{i i}^{\mathrm{eff}} \equiv \frac{\rho v_{\mathrm{th}}^{2}}{2 \eta_{0}}
$$

The coefficient governing the diffusion of species 2 into species 1 (e.g., helium into hydrogen) is given by 

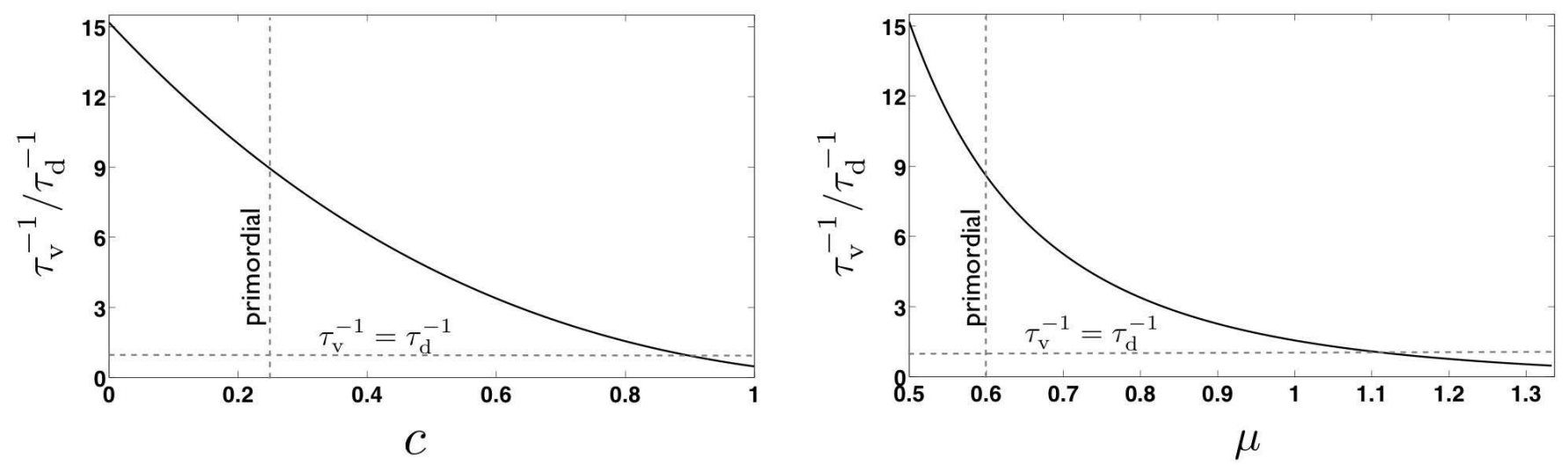

Figure 7. Ratio of the inverse timescales associated with viscous and diffusion processes for a binary mixture of $\mathrm{H}$ and He as a function of the composition $c$ (left) and mean molecular weight $\mu$ (right). The vertical dashed line indicates the values for which the mixture has primordial composition, i.e., $c \simeq 0.25$, which corresponds to $\mu \simeq 0.6$.

\section{(Bahcall \& Loeb 1990)}

$$
\begin{aligned}
D= & \frac{3}{4 \sqrt{2 \pi}} \frac{m_{2}\left(k_{B} T\right)^{5 / 2}}{\sqrt{m_{12}} q_{1}^{2} q_{2}^{2} \rho \ln \Lambda_{12}}\left[\frac{4-c}{(2-c)(8-5 c)}\right] \\
& (0<c<1) .
\end{aligned}
$$

Therefore the ratio $\tau_{\mathrm{v}}^{-1} / \tau_{\mathrm{d}}^{-1}=3 \nu_{\|} / D$ is given by

$$
\begin{aligned}
& \frac{\tau_{\mathrm{v}}^{-1}}{\tau_{\mathrm{d}}^{-1}} \simeq 3 \sqrt{\frac{2}{5}}\left[\frac{(2-c)(8-5 c)}{4-c}\right] \\
& \times\left[\frac{2}{1+\sqrt{\frac{8}{5}}\left(\frac{c}{1-c}\right)}+\frac{0.25}{1+\sqrt{\frac{2}{5}}\left(\frac{1-c}{c}\right)}\right],
\end{aligned}
$$

which is shown in Figure 7 as a function of the concentration $c$ (left panel) and as a function of the mean molecular weight $\mu$ (right panel). In the inner regions of the ICM, where $c \simeq 0.6$ or $\mu \simeq 0.8$ (see Figure 5), viscous and diffusion processes take place on comparable timescales, i.e., $\tau_{\mathrm{v}}^{-1} / \tau_{\mathrm{d}}^{-1} \simeq 3$, while $\tau_{\mathrm{v}}^{-1} / \tau_{\mathrm{d}}^{-1} \simeq 9$ for a primordial mixture of helium and hydrogen, i.e., $c \simeq 0.25$ or $\mu \simeq 0.6$. It should be kept in mind that in carrying out this calculation we have assumed that all the ratios between the Coulomb logarithms are of order unity and the expressions for the transport coefficients along the magnetic field lines are identical to the ones that are valid in the absence of the magnetic field.

\section{REFERENCES}

Abramopoulos, F., Chanan, G. A., \& Ku, W. H.-M. 1981, ApJ, 248, 429

Bahcall, J. N., \& Loeb, A. 1990, ApJ, 360, 267

Balbus, S. A. 2000, ApJ, 534, 420

-. 2001, ApJ, 562, 909

-. 2004, ApJ, 616, 857

Balbus, S. A., \& Reynolds, C. S. 2010, ApJ, 720, L97

Bogdanović, T., Reynolds, C. S., Balbus, S. A., \& Parrish, I. J. 2009, ApJ, 704, 211

Braginskii, S. I. 1965, Reviews of Plasma Physics, 1, 205

Bulbul, G. E., Hasler, N., Bonamente, M., \& Joy, M. 2010, ApJ, 720, 1038

Bulbul, G. E., Hasler, N., Bonamente, M., Joy, M., Marrone, D., Miller, A., \& Mroczkowski, T. 2011, A\&A, 533, A6

Carilli, C. L., \& Taylor, G. B. 2002, ARA\&A, 40, 319
Cavagnolo, K. W., Donahue, M., Voit, G. M., \& Sun, M. 2009, ApJS, 182, 12

Chandran, B. D., \& Dennis, T. J. 2006, ApJ, 642, 140

Chew, G. F., Goldberger, M. L., \& Low, F. E. 1956, Royal Society of London Proceedings Series A, 236, 112

Chuzhoy, L., \& Loeb, A. 2004, MNRAS, 349, L13

Chuzhoy, L., \& Nusser, A. 2003, MNRAS, 342, L5

Ettori, S., \& Fabian, A. C. 2006, MNRAS, 369, L42

Fabian, A. C., \& Pringle, J. E. 1977, MNRAS, 181, 5P

Gilfanov, M. R., \& Syunyaev, R. A. 1984, Soviet Astronomy Letters, 10, 137

Hollweg, J. V. 1985, J. Geophys. Res., 90, 7620

Kunz, M. W. 2011, MNRAS, 417, 602

Kunz, M. W., Bogdanović, T., Reynolds, C. S., \& Stone, J. M. 2012, ApJ, 754,122

Landau, L. D., \& Lifshitz, E. M. 1959, Fluid mechanics

Latter, H. N., \& Kunz, M. W. 2012, MNRAS, 423, 1964

Leccardi, A., \& Molendi, S. 2008, A\&A, 486, 359

Markevitch, M. 2007, ArXiv e-prints

McCourt, M., Parrish, I. J., Sharma, P., \& Quataert, E. 2011, MNRAS, 413, 1295

McCourt, M., Sharma, P., Quataert, E., \& Parrish, I. J. 2012, MNRAS, 419, 3319

Narayan, R., \& Medvedev, M. V. 2001, ApJ, 562, L129

Parrish, I. J., McCourt, M., Quataert, E., \& Sharma, P. 2012, MNRAS, 422, 704

Parrish, I. J., \& Quataert, E. 2008, ApJ, 677, L9

Parrish, I. J., Quataert, E., \& Sharma, P. 2009, ApJ, 703, 96

-. 2010, ApJ, 712, L194

Parrish, I. J., \& Stone, J. M. 2005, ApJ, 633, 334

-. 2007, ApJ, 664, 135

Parrish, I. J., Stone, J. M., \& Lemaster, N. 2008, ApJ, 688, 905

Peng, F., \& Nagai, D. 2009, ApJ, 693, 839

Piffaretti, R., Jetzer, P., Kaastra, J. S., \& Tamura, T. 2005, A\&A, 433, 101

Qin, B., \& Wu, X.-P. 2000, ApJ, 529, L1

Quataert, E. 2008, ApJ, 673, 758

Ruszkowski, M., \& Oh, S. P. 2010, ApJ, 713, 1332

Schekochihin, A. A., Cowley, S. C., Kulsrud, R. M., Hammett, G. W., \& Sharma, P. 2005, ApJ, 629, 139

Schekochihin, A. A., Cowley, S. C., Kulsrud, R. M., Rosin, M. S., \&

Heinemann, T. 2008, Physical Review Letters, 100, 081301

Sharma, P., Hammett, G. W., \& Quataert, E. 2003, ApJ, 596, 1121

Sharma, P., Parrish, I. J., \& Quataert, E. 2010, ApJ, 720, 652

Shtykovskiy, P., \& Gilfanov, M. 2010, MNRAS, 401, 1360

Snyder, P. B., Hammett, G. W., \& Dorland, W. 1997, Physics of Plasmas, 4, 3974

Spitzer, L. 1962, Physics of Fully Ionized Gases

Tamura, T., Kaastra, J. S., den Herder, J. W. A., Bleeker, J. A. M., \&

Peterson, J. R. 2004, A\&A, 420, 135

Vikhlinin, A., Kravtsov, A., Forman, W., Jones, C., Markevitch, M., Murray, S. S., \& Van Speybroeck, L. 2006, ApJ, 640, 691 